\title{
Photoactivation of the Cytotoxic Properties of \\ Platinum(II) Complexes through Ligand
}

\section{Photoswitching}

Andreu Presa, ${ }^{\dagger}$ Guillem Vázquez, ${ }^{\dagger}$ Leoní A. Barrios, ${ }^{\dagger}$ Olivier Roubeau, ${ }^{\ddagger}$ Luís Korrodi-Gregório, ${ }^{\S}$ Ricardo Pérez-Tomás, ${ }^{\S}$ and Patrick Gamez* ${ }^{* \dagger}$.

† Department of Inorganic and Organic Chemistry, Inorganic Chemistry Section, University of Barcelona, Martí i Franquès 1-11, 08028 Barcelona, Spain.

‡ Instituto de Ciencia de Materiales de Aragón (ICMA), CSIC and Universidad de Zaragoza, Plaza San Francisco s/n, 50009 Zaragoza, Spain.

$\S$ Department of Pathology and Experimental Therapeutics, Faculty of Medicine, University of Barcelona, Campus Bellvitge, Feixa Llarga s/n, 08907 L'Hospitalet de Llobregat, Spain.

\| Catalan Institution for Research and Advanced Studies (ICREA), Passeig Lluís Companys 23, 08010 Barcelona, Spain.

$\perp$ Institute of Nanoscience and Nanotechnology (IN2UB), Universitat de Barcelona.

E-mail: patrick.gamez@qi.ub.es 


\section{KEYWORDS}

Photochemistry - Photoactivation - Platinum - Anticancer - Photoactivated chemotherapy Diarylethenes.

\section{ABSTRACT}

The development of photoactivatable metal complexes with potential anticancer properties is a topical area of current investigation. Photoactivated chemotherapy (PACT) using coordination compounds is typically based on photochemical processes occurring at the metal center. In the present study, an innovative approach is applied that takes advantage of the remarkable photochemical properties of diarylethenes. Following a proof-of-concept study with two complexes, namely $\mathbf{C 1}$ and $\mathbf{C 2}{ }^{1}$ a series of additional platinum(II) complexes from dithienylcyclopentene-based ligands have been designed and prepared. Like $\mathbf{C 1}$ and $\mathbf{C 2}$, these new coordination compounds exhibit two thermally stable, interconvertible photoisomers that display distinct properties. The photochemical behavior of ligands $\mathbf{L} 3-\mathbf{L} 7$ has been analyzed by ${ }^{1} \mathrm{H}$ NMR and UV-vis spectroscopy. Subsequently, the corresponding platinum(II) complexes C3-C7 have been synthesized and fully characterized, including by X-ray diffraction for some of them. Next, the interaction of each photoisomer (i.e., containing the open or closed ligand) of the metal complexes with DNA has been examined thoroughly using various techniques, revealing their distinct DNA-binding modes and affinities, as observed for the earlier compounds $\mathbf{C 1}$ and $\mathbf{C 2}$. The antiproliferative activity of the two forms of the complexes has then been assessed with five cancer 
cell lines and compared with that of $\mathbf{C 1}$ and $\mathbf{C 2}$, which supported the use of such diarylethenebased systems for the generation of a new class of potential photochemotherapeutic metallodrugs.

\section{INTRODUCTION}

An elegant drug-design approach aimed at enhancing tumor selectivity while reducing systemic toxicity is the use of light activation. ${ }^{2}$ Recent advances in laser and fiber-optic technologies have driven the development of various medical applications based on light, ${ }^{3}$ including the design of metal-containing prodrugs that can be activated through irradiation. ${ }^{2}, 4$ Photoactivated chemotherapy (PACT) is a prominent field of investigation, which provides both temporal and spatial control over drug activity, and offers tremendous potential for the treatment of different types of cancer. ${ }^{5}$

Photoactivatable prodrugs based on coordination compounds typically involve activation at the metal center, with concomitant generation of pharmacologically active species. ${ }^{6}$ Metalmediated PACT can be achieved via distinct mechanisms of action, namely photodissociation ${ }^{7}$ and photosensitisation, ${ }^{8}$ which are often associated to the redox properties of the metal center, ${ }^{2}$ and photothermal reaction. ${ }^{9}$ A number of research groups worldwide are developing elegant systems that are photoactivatable through the action of light on the metal. ${ }^{10-17}$

The activation of a metallodrug through the photomodification of a coordinated ligand has not been investigated extensively. Different types of organic molecular photoswitches may be used for this purpose, like azobenzenes, spiropyrans or diarylethenes. ${ }^{18}$ Azobenzenes and spiropyrans

are thermally unstable and gradually revert back to their initial state in the absence of light. ${ }^{19}$ In 
contrast, diarylethenes are more suitable for such biological application as they exhibit negligible thermal relaxation; as a matter of fact, these unique properties have converted them as one of the most interesting classes of photoresponsive molecular devices, as reflected by their numerous applications. ${ }^{20}$

In the present study, the potential use of diarylethene moieties to generate potential lightactivatable metallodrugs has been investigated further. ${ }^{1}$ Indeed, it has recently been shown that the two thermally stable states of two molecular switches (complexes $\mathbf{C} 1$ and $\mathbf{C} 2$ in Figure 1) exhibit distinct biological activities, one of the two photoisomers being cytotoxic whereas the other one not. ${ }^{1}$ Hence, based on these results, a series of new simple diarylethene-based ligands were designed and prepared, and the corresponding platinum(II) complexes were synthesized. Subsequently, the DNA-interacting properties of their open and closed forms were examined using various techniques, and their cytotoxic behavior against various cancer cell lines was then evaluated and compared with those of $\mathbf{C 1}$ and C2. Promising results were achieved, further indicating that this innovative mechanism of photochemical control may be applied to produce a new class of photoactivatable metallodrugs. It can indeed be stressed that the photoactivation of the cytotoxic properties of the platinum(II) compounds reported herein is based on the photomodification of the coordinated ligand, and not on the metal center (as commonly described in the literature). 
A<smiles>[R]C1=CC2=C3CC[R8]C3=C3C=C([R7])S[C@]3(C)C2=C1</smiles><smiles>Cc1sc(-c2cc[n+](C(Cl)(Cl)Cl)cc2)cc1C1=C(c2cc(-c3cc[n+](C(Cl)(Cl)P(C)(C)=O)cc3)sc2C)CCC1</smiles>
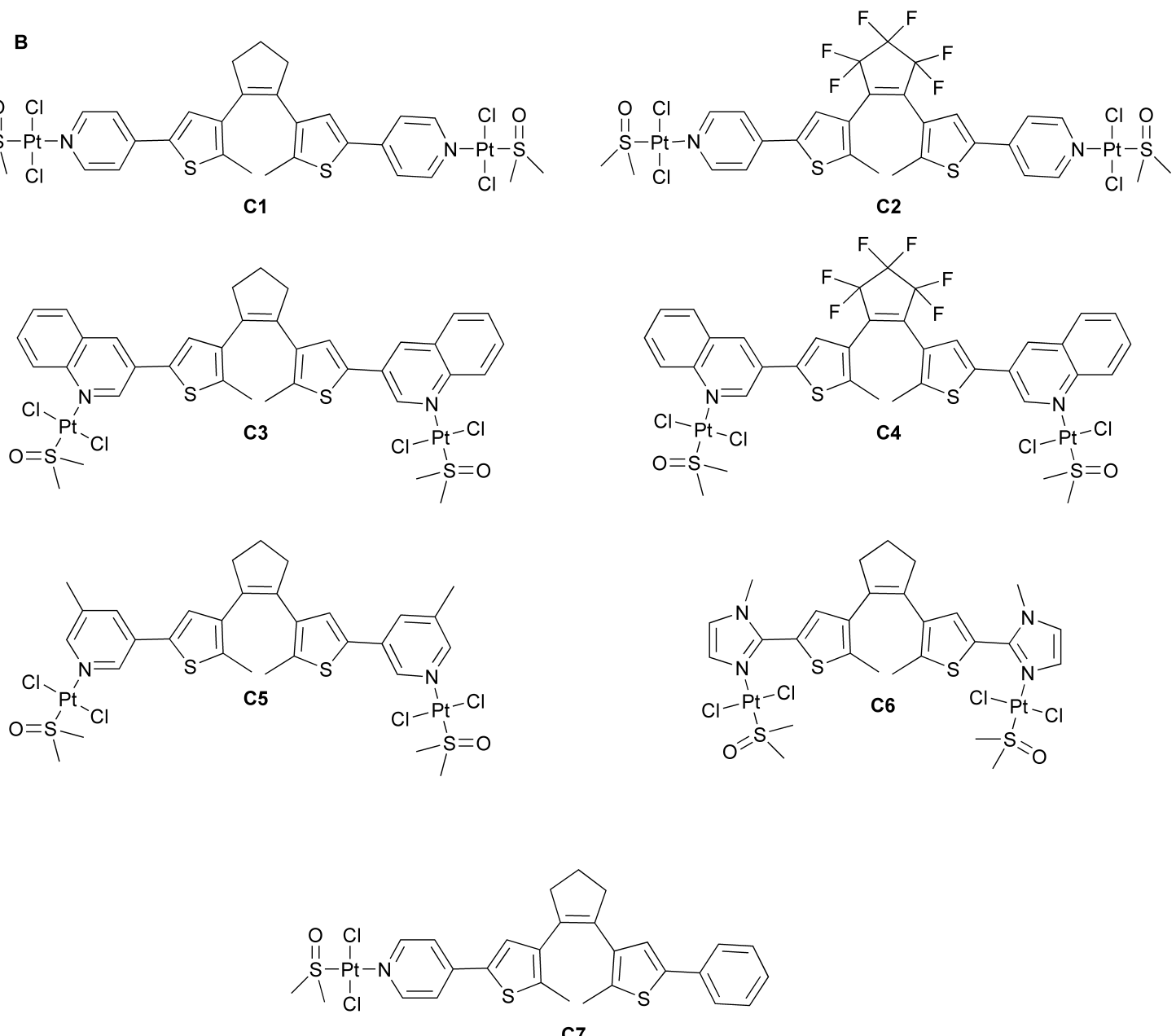

Figure 1. A) Reversible photocyclization of dithienylcyclopentenes $(\mathrm{R}=\mathrm{H})$ and dithienylperfluorocyclopentenes $(\mathrm{R}=\mathrm{F})$; B) Schematic representation of the platinum(II) complexes C1-C7, obtained with seven different Dithienylcyclopentene-based ligands. 


\section{EXPERIMENTAL DETAILS}

Materials and methods. All reagents and HPLC-grade solvents for the synthesis of the ligands and complexes were purchased from commercial sources and used as received. Ethidium bromide, sodium cacodylate, TAE (Tris Acetate-EDTA) and calf thymus DNA (ct-DNA) were purchased from Sigma-Aldrich. Plasmid pBR322 DNA was purchased from Roche. All reagents used for the in vitro DNA-interaction studies were obtained from Sigma-Aldrich and Invitrogen. Anhydrous solvents were distilled under an inert atmosphere using a PureSolv ${ }^{\mathrm{TM}}$ solvent purification system from Innovative Technologies.

When required, the reactions were performed under an atmosphere of dinitrogen using standard procedures. Column chromatography purifications were carried out in air, using ultrapure silica gel $(60-200 \mu \mathrm{m}, 60 \AA)$ from Acros Organics, and monitored by analytical thin-layer chromatography (TLC) using pre-coated aluminum plates.

Nuclear magnetic resonance (NMR) spectra were recorded at 298 K on a Varian Mercury 400 $\mathrm{MHz}$ spectrometer. Chemical shifts $(\delta)$ are reported in ppm and coupling constants $(J)$ are given in Hz. Proton chemical shifts are referenced to the corresponding non-deuterated solvent peak ( $\mathrm{CHCl}_{3}$ : $7.26 \mathrm{ppm}$; DMSO: $2.50 \mathrm{ppm}$ ); fluorine chemical shifts are referenced to trifluoroacetic acid (-76.55 ppm). Signal multiplicities are defined as $\mathrm{s}$ (singlet), $\mathrm{d}$ (doublet), $\mathrm{t}$ (triplet), $\mathrm{q}$ (quartet), quint (quintet), br (broad signal) and m (multiplet).

ESI mass spectrometry was carried out using a LC/MSD-TOF spectrometer from Agilent Technologies equipped with an electrospray ionization (ESI) source, at the Centres Cientifics i Tecnologics de la Universitat de Barcelona. Samples were eluted with a $\mathrm{H}_{2} \mathrm{O} / \mathrm{CH}_{3} \mathrm{CN}$ 1:1 mixture and measured in the positive mode. $\mathrm{C}, \mathrm{H}, \mathrm{N}$ and $\mathrm{S}$ elemental analyses were performed at the 
Centres Cientifics i Tecnologics de la Universitat de Barcelona, using a Thermo EA 1108 CHNS/O analyzer from Carlo Erba Instruments.

Spectroscopic measurements in buffered aqueous media were performed in cacodylate buffer solution ( $1 \mathrm{mM}$ sodium cacodylate, $20 \mathrm{mM} \mathrm{NaCl}, \mathrm{pH}=7.2$ ), which was prepared with ultrapure water and whose $\mathrm{pH}$ was adjusted with $\mathrm{HCl}_{\mathrm{aq}}$. The $\mathrm{UV}$ and visible irradiation of the samples was carried out with a MAX-303 light source from Asahi Spectra, using appropriate bandpass filters. The UV-Vis absorption spectra were recorded on a Varian Cary 100 Bio spectrophotometer.

Fluorescence and circular dichroism spectra were collected in $1 \mathrm{~cm}$-pathlength quartz cuvettes, using cacodylate buffered solutions.

The stock solutions for the DNA-interaction studies were prepared as follows: the commercial plasmid pBR322 stock solution $\left(250 \mu \mathrm{g} \mathrm{mL}-1\right.$, ca. $\left.385 \mu \mathrm{M}_{\mathrm{bp}}\right)$ was used as received. A $150 \mu \mathrm{M}$ stock solution of ct-DNA was prepared by dissolving the highly-polymerized sodium salt of the biomolecule in cacodylate buffer, and the exact concentration was determined by absorbance at $260 \mathrm{~nm}\left(\varepsilon_{\mathrm{bp}}=13200 \mathrm{M}^{-1} \mathrm{~cm}^{-1}\right) .5 \mathrm{mM}$ stock solutions of the studied complexes were freshly prepared in DMSO before each experiment, and further diluted in DMSO/buffer mixtures as required. Stock solutions of closed complexes were obtained by irradiation of the corresponding solutions of the open complexes, at $\lambda<365 \mathrm{~nm}$, until reaching the photostationary state (PSS).

\section{Synthesis}

1,5-Bis(5-chloro-2-methyl-3-thienyl)pentane-1,5-dione (1). ${ }^{21}$ Aluminum(III) trichloride (29.0 g, $217 \mathrm{mmol}$ ) was slowly added to a mixture of 2-chloro-5-methylthiophene (25.0 g, $189 \mathrm{mmol}$ ) and glutaryl dichloride (12.0 mL, $94 \mathrm{mmol})$ dissolved in $200 \mathrm{~mL}$ of ice-cold nitromethane. After the addition of $\mathrm{AlCl}_{3}$, the resulting dark red solution was stirred for $3 \mathrm{~h}$ at room temperature and $150 \mathrm{~mL}$ of ice-cold water were subsequently added in small portions. The reaction mixture was 
then placed in an ice bath and vigorously stirred for $1 \mathrm{~h}$, until an abundant precipitate formed. This precipitate was then poured over a glass filter, washed with cold $n$-pentane and finally dried under reduced pressure to yield the crude final product $\mathbf{1}$ as a pale brown solid (27.6 g, 81\%).

${ }^{1} \mathrm{H}$ NMR (400 MHz, $\mathrm{CDCl}_{3}$ ): $\delta=2.06\left(\mathrm{~m}, 2 \mathrm{H}, J=6.8 \mathrm{~Hz}, \mathrm{CH}_{2}\right.$ ), 2.66 (s, 6H, Me), 2.86 (t, 4H, $J=6.8 \mathrm{~Hz}, \mathrm{COCH}_{2}$ ), 7.18 (s, 2H, thiophene). MS (ESI): $\mathrm{m} / \mathrm{z}=361[\mathrm{M}]^{+}$(expected: 360.98). Elemental analyses calculated for $\mathrm{C}_{15} \mathrm{H}_{14} \mathrm{Cl}_{2} \mathrm{O}_{2} \mathrm{~S}_{2}$ (\%): C 49.86, H 3.91, S 17.75; found: C 50.19, H 3.94, S 16.56.

1,2-Bis(5-chloro-2-methyl-3-thienyl)cyclopentene (2). Titanium(IV) tetrachloride (4.4 mL, 40 mmol) was carefully added under a nitrogen atmosphere using a syringe to an ice-cooled suspension of zinc dust $(5.2 \mathrm{~g}, 80 \mathrm{mmol})$ in $150 \mathrm{~mL}$ of anhydrous THF. The resulting grey-blue reaction mixture was slowly heated to $50{ }^{\circ} \mathrm{C}$ and stirred for $2 \mathrm{~h}$, after which pyridine ( $3.2 \mathrm{~mL}, 40$ mmol) was added dropwise, producing a brown solution. After 10 min, 1 (7.2 g, 20 mmol) was added and the resulting mixture was stirred in the dark overnight. Next, the solution was cooled in an ice bath and quenched with a $20 \%$ aqueous $\mathrm{K}_{2} \mathrm{CO}_{3}$ solution ( $25 \mathrm{~mL}$ ), yielding an abundant black precipitate. After vigorous stirring of the precipitate with diethyl ether $(40 \mathrm{~mL})$, the remaining solid was isolated by filtration over a glass filter and washed with additional diethyl ether $(2 \times 25$ $\mathrm{mL}$ ). The combined organic phase was placed in an ice bath for 30 min and filtered again to eliminate the resulting undesired white precipitate. The solution was washed with acidified water ( $2 \times 20 \mathrm{~mL}$ ), dried over $\mathrm{Na}_{2} \mathrm{SO}_{4}$, filtered and concentrated under reduced pressure to give an orange oil. This oil was purified by column chromatography on silica gel with cyclohexane as the eluent, yielding an oil that slowly solidified to colorless product 2 (4.75 g, 72\%).

${ }^{1} \mathrm{H}$ NMR (400 MHz, $\mathrm{CDCl}_{3}$ ): $\delta=1.88$ (s, 6H, Me), 2.02 (m, 2H, $J=7.6 \mathrm{~Hz}$, cyclopentene), 2.71 (t, 4H, $J=7.6 \mathrm{~Hz}$, cyclopentene), 6.57 (s, 2H, thiophene). MS (ESI): m/z = $329[\mathrm{M}]^{+}$(expected: 
328.99). Elemental Analyses calculated for $\mathrm{C}_{15} \mathrm{H}_{14} \mathrm{Cl}_{2} \mathrm{~S}_{2}$ (\%): C 54.71, H 4.29, S 19.47; found: C 54.89, H 4.37, S 18.78.

4-Bromo-5-methylthiophene-2-boronic acid (3). $n$-Buthyllithium (10 mL of a $1.6 \mathrm{M}$ solution in hexane, $16.0 \mathrm{mmol})$ was added dropwise to a solution of 3,5-dibromo-2-methylthiophene (2.0 $\mathrm{mL}, 15.6 \mathrm{mmol})$ in anhydrous diethyl ether $(25 \mathrm{~mL})$ at $-78^{\circ} \mathrm{C}$, under a dinitrogen atmosphere, resulting in a colour change from colorless to bright orange. The reaction mixture was stirred for $30 \mathrm{~min}$, and then tributyl borate $(8.0 \mathrm{~mL}, 29.6 \mathrm{mmol})$ was added. After stirring the resulting solution at room temperature overnight, $5 \% \mathrm{HCl}$ aqueous solution $(20 \mathrm{~mL})$ was added to redissolve the pale-yellow precipitate formed. The organic phase was then separated and extracted with an aqueous $\mathrm{NaOH}$ solution (1 M, $2 \times 20 \mathrm{~mL}$ ); the combined aqueous phase was acidified ( $\mathrm{HCl}, 37 \%)$ under vigorous stirring until the apparition of an off-white precipitate. The product $\mathbf{3}$ was then filtered, washed with acidified water and dried under reduced pressure (3.3 g, 96\%).

${ }^{1} \mathrm{H}$ NMR (400 MHz, DMSO-d $\left.\mathrm{d}_{6}\right): \delta=2.36$ (s, 3H, Me), 7.49 (s, 1H, thiophene), 8.32 (s, 2H, $\left.\mathrm{B}(\mathrm{OH})_{2}\right)$. MS (ESI): m/z = $220.9[\mathrm{M}]^{+}$(expected: 220.94).

4-Bromo-5-methyl-2-(4-pyridyl)thiophene (4). 4-Bromopyridine hydrochloride (1.3 g, 6.7 mmol) and 3 (1.0 g, $4.5 \mathrm{mmol}$ ) were subsequently added under a nitrogen atmosphere to a solution of $\left[\mathrm{Pd}\left(\mathrm{PPh}_{3}\right)_{4}\right](260 \mathrm{mg}, 5 \% \mathrm{~mol})$ in anhydrous THF $(20 \mathrm{~mL})$ and $20 \%$ aqueous $\mathrm{K}_{2} \mathrm{CO}_{3}(20 \mathrm{~mL})$. The resulting two-phase system was heated to $50{ }^{\circ} \mathrm{C}$, stirred in the dark overnight and then cooled to room temperature before dichloromethane $(25 \mathrm{~mL})$ and water $(15 \mathrm{~mL})$ were added. The organic layer was separated, washed with brine $(25 \mathrm{~mL})$, dried over $\mathrm{Na}_{2} \mathrm{SO}_{4}$, filtered and concentrated under reduced pressure. The crude product was purified by column chromatography on silica gel with dichloromethane as the eluent, yielding the final product 4 as an off-white solid (0.94 g, 82\%). 
${ }^{1} \mathrm{H}$ NMR (400 MHz, $\mathrm{CDCl}_{3}$ ): $\delta=2.45$ (s, 3H, Me), 7.32 (s, 1H, thiophene), 7.38 (m, 2H, $J_{1}=$ $6.0 \mathrm{~Hz}, J_{2}=2.4 \mathrm{~Hz}$, pyridine), $8.59\left(\mathrm{~m}, 2 \mathrm{H}, J_{1}=6.0 \mathrm{~Hz}, J_{2}=2.4 \mathrm{~Hz}\right.$, pyridine). MS (ESI): m/z = $254.0[\mathrm{M}]^{+}$(expected: 253.96).

4-Bromo-5-methyl-2-(3-quinolyl)thiophene (5). Compound 5 was prepared following the procedure described for compound 4, but using 3-bromoquinoline ( $0.9 \mathrm{~mL}, 6.7 \mathrm{mmol})$ instead of 4-bromopyridine. The crude product was purified by column chromatography on silica gel with dichloromethane as the eluent, to yield the final product as an off-white solid (0.81 g, 59\%).

${ }^{1} \mathrm{H}$ NMR (400 MHz, $\mathrm{CDCl}_{3}$ ): $\delta=2.48$ (s, 3H, Me), 7.31 (s, 1H, thiophene), 7.58 (m, 2H, quinoline), 7.71 (m, 2H, quinoline), 7.84 (d, 2H, $J=8.8 \mathrm{~Hz}$, quinoline), 8.12 (d, $2 \mathrm{H}, J=8.8 \mathrm{~Hz}$, quinoline), 8.21 (d, 2H, $J=2.4 \mathrm{~Hz}$, quinoline), 9.11 (d, 2H, $J=2.4 \mathrm{~Hz}$, quinoline). MS (ESI): m/z $=306.0[\mathrm{M}+\mathrm{H}]^{+}$(expected: 305.97).

1-(2-Methyl-5-chloro-3-thienyl)-2-(2-methyl-5-phenyl-3-thienyl)-cyclopentene (6). 2 (1.0 g, $3.0 \mathrm{mmol})$ was dissolved in anhydrous THF (10 mL) under a nitrogen atmosphere and treated with n-buthyllithium (2.0 mL of a $1.6 \mathrm{M}$ solution in hexane, $3.2 \mathrm{mmol})$ at room temperature. After stirring in the dark for about $45 \mathrm{~min}$, tributyl borate $(1.2 \mathrm{~mL}, 4.5 \mathrm{mmol})$ was added and the resulting bright orange solution was then stirred in the dark for $1 \mathrm{~h}$; it was subsequently used for the Suzuki cross-coupling reaction. In the meantime, in a separate flask, $\left[\mathrm{Pd}\left(\mathrm{PPh}_{3}\right)_{4}\right](180 \mathrm{mg}, 5 \% \mathrm{~mol})$ and bromobenzene $(0.4 \mathrm{~mL}, 3.75 \mathrm{mmol})$ were dissolved in a solvent mixture containing anhydrous THF (20 mL) and 20\% aqueous $\mathrm{K}_{2} \mathrm{CO}_{3}$ solution (20 mL), under a dinitrogen atmosphere. This two-phase system was stirred at $50{ }^{\circ} \mathrm{C}$ for $15 \mathrm{~min}$ and the previous freshly prepared boronic derivative solution was added dropwise with a syringe. The resulting reaction mixture was stirred in the dark overnight, after which it was cooled to room temperature; dichloromethane (25 mL) and water $(15 \mathrm{~mL})$ were subsequently added. The organic layer was separated, washed with brine 
(25 mL), dried over $\mathrm{Na}_{2} \mathrm{SO}_{4}$, filtered and concentrated under reduced pressure. The crude product was purified by column chromatography on silica gel with a 19:1 mixture of cyclohexane and ethyl acetate as the eluent, yielding 6 as a slightly colored oil ( 0.74 g, 66\%).

${ }^{1} \mathrm{H}$ NMR (400 MHz, CDCl $): \delta=1.88$ (s, 3H, Me), 2.00 (s, 3H, Me), 2.05 (m, 2H, 7.6 Hz, cyclopentene), $2.75\left(\mathrm{~m}, 2 \mathrm{H}, J_{\mathrm{app}}=7.6 \mathrm{~Hz}\right.$, cyclopentene), $2.81\left(\mathrm{~m}, 2 \mathrm{H}, J_{\mathrm{app}}=7.6 \mathrm{~Hz}\right.$, cyclopentene), 6.62 (s, $1 \mathrm{H}$, thiophene), 6.99 (s, $1 \mathrm{H}$, thiophene), 7.23 (m, $1 \mathrm{H}, J_{\text {app }}=7.2 \mathrm{~Hz}$, phenyl), 7.34 (m, 2H, $J_{\text {app }}=7.2 \mathrm{~Hz}$, phenyl), 7.49 (m, 2H, $J_{\text {app }}=7.2 \mathrm{~Hz}$, phenyl). MS (ESI): m/z = 371.1 $[\mathrm{M}]^{+}$(expected: 371.07).

1,2-Bis(2-methyl-5-(4-pyridyl)-3-thienyl)cyclopentene (L1). Ligand L1, whose synthetic pathway was described earlier, ${ }^{1}$ was prepared as described below. This procedure was applied for all ligands including a central cyclopentene ring (Ligands L3, L5, L6 and L7).

$2(1.0 \mathrm{~g}, 3.0 \mathrm{mmol})$ was dissolved in anhydrous THF $(10 \mathrm{~mL})$ under a nitrogen atmosphere and treated with $n$-buthyllithium (4.0 $\mathrm{mL}$ of a $1.6 \mathrm{M}$ solution in hexane, $6.4 \mathrm{mmol}$ ) at room temperature. The reaction mixture was stirred in the dark for about 45 min until a light brown precipitate appeared, and tributyl borate $(2.4 \mathrm{~mL}, 8.9 \mathrm{mmol})$ was added subsequently. The resulting bright orange solution was stirred in the dark for $1 \mathrm{~h}$ and subsequently used for a Suzuki cross-coupling reaction. Hence, in a separate flask, $\left[\mathrm{Pd}\left(\mathrm{PPh}_{3}\right)_{4}\right](360 \mathrm{mg}, 5 \% \mathrm{~mol})$ and 4bromopyridine hydrochloride (1.46 g, $7.5 \mathrm{mmol}$ ) were dissolved in a mixture of anhydrous THF (20 mL) and $20 \%$ aqueous solution of $\mathrm{K}_{2} \mathrm{CO}_{3}(20 \mathrm{~mL})$ under a nitrogen atmosphere. This twophase system was stirred at $50{ }^{\circ} \mathrm{C}$ for 15 min and the previous freshly prepared solution of the boronic derivative of $\mathbf{2}$ was added dropwise with a syringe. The resulting reaction mixture was then stirred in the dark overnight, after which it was cooled to room temperature and treated with dichloromethane $(25 \mathrm{~mL})$ and water $(15 \mathrm{~mL})$. The organic layer was separated, washed with brine 
(25 mL), dried over $\mathrm{Na}_{2} \mathrm{SO}_{4}$, filtered and concentrated under reduced pressure. The crude product was purified by column chromatography on silica gel with ethyl acetate (containing $1 \% \mathrm{NH}_{3}$ ) as the eluent, yielding the final product as a white solid ( $0.60 \mathrm{~g}, 48 \%)$.

${ }^{1} \mathrm{H}$ NMR (400 MHz, $\mathrm{CDCl}_{3}$ ): $\delta=2.03$ (s, 6H, Me), 2.11 (m, 2H, $J=7.6 \mathrm{~Hz}$, cyclopentene), 2.85 (t, 4H, $J=7.6 \mathrm{~Hz}$, cyclopentene), 7.22 (s, 2H, thiophene), 7.35 (m, 4H, $J_{\text {app }}=6.4 \mathrm{~Hz}$, pyridine), $8.53\left(\mathrm{~m}, 4 \mathrm{H}, J_{\text {app }}=6.4 \mathrm{~Hz}\right.$, pyridine). ${ }^{13} \mathrm{C}$ NMR (100 MHz, $\left.\mathrm{CDCl}_{3}\right): \delta=14.8,23.1,38.6,119.4$, 126.4, 134.9, 136.8, 137.2, 137.4, 141.4, 150.4. MS (ESI): $\mathrm{m} / \mathrm{z}=415.1[\mathrm{M}+\mathrm{H}]^{+}$(expected: 415.13). Elemental Analyses calculated for $\mathrm{C}_{25} \mathrm{H}_{22} \mathrm{~N}_{2} \mathrm{~S}_{2}$ (\%): C 72.43, H 5.35, N 6.76, S 15.47; found: C 72.75, H 5.40, N 6.59, S 15.30.

1,2-Bis(2-methyl-5-(4-pyridyl)-3-thienyl)perfluorocyclopentene (L2). Ligand L2, whose synthetic pathway was described earlier, ${ }^{1}$ was prepared as described below. This procedure was applied for the other ligand containing a central perfluorocyclopentene ring, namely $\mathbf{L} 4$.

$4(1.0 \mathrm{~g}, 4.0 \mathrm{mmol})$ was suspended in anhydrous diethyl ether $(15 \mathrm{~mL})$ under a nitrogen atmosphere. Then, $n$-buthyllithium (2.6 $\mathrm{mL}$ of a $1.6 \mathrm{M}$ solution in hexane, $4.2 \mathrm{mmol}$ ) was added dropwise at $-78^{\circ} \mathrm{C}$. After stirring the resulting pink suspension for $30 \mathrm{~min}$, octafluorocyclopentene (1.35 $\mathrm{mL}$ of a $20 \%$ solution in anhydrous $\mathrm{Et}_{2} \mathrm{O}, 2.0 \mathrm{mmol}$ ) was added and the ensuing reaction mixture was stirred for $1 \mathrm{~h}$ at $-78^{\circ} \mathrm{C}$. The cooling bath was subsequently removed, and the stirring continued at room temperature for $3 \mathrm{~h}$, after which dichloromethane $(25 \mathrm{~mL})$ and water $(25 \mathrm{~mL})$ were added. The organic layer was separated, washed with brine ( $25 \mathrm{~mL})$, dried over $\mathrm{Na}_{2} \mathrm{SO}_{4}$, filtered and concentrated under reduced pressure. The crude product was purified by column chromatography on silica gel using ethyl acetate (containing $1 \% \mathrm{NH}_{3}$ ) as the eluent, yielding $\mathbf{L} 2$ as a pale brown solid $(0.27 \mathrm{~g}, 26 \%)$. The low yield may be due to the fact that octafluorocyclopentene is highly volatile (B.p. $=27^{\circ} \mathrm{C}$ ). 
${ }^{1} \mathrm{H}$ NMR (400 MHz, $\left.\mathrm{CDCl}_{3}\right): \delta=2.00(\mathrm{~s}, 6 \mathrm{H}, \mathrm{Me}), 7.41\left(\mathrm{~m}, 4 \mathrm{H}, J_{1}=4.4 \mathrm{~Hz}, J_{2}=1.6 \mathrm{~Hz}\right.$, pyridine), 7.47 (s, 2H, thiophene), 8.61 (m, 4H, $J_{1}=4.4 \mathrm{~Hz}, J_{2}=1.6 \mathrm{~Hz}$, pyridine). ${ }^{19} \mathrm{~F}$ NMR (400 $\mathrm{MHz}, \mathrm{CDCl}_{3}$ ): $\delta=-131.81$ (quint, $2 \mathrm{H}, J=5.6 \mathrm{~Hz}$ ), -110.11 (t, 4H, $\left.J=5.6 \mathrm{~Hz}\right) .{ }^{13} \mathrm{C}$ NMR $(100$ $\mathrm{MHz}, \mathrm{CDCl}_{3}$ ): $\delta=14.9,111.0$ (multiplet), 116.1 (multiplet), 119.7, 124.8, 126.3, 136.3 (multiplet), 139.5, 140.3, 143.8, 150.7. MS (ESI): $\mathrm{m} / \mathrm{z}=523.1[\mathrm{M}+\mathrm{H}]^{+}$(expected: 523.07). Elemental Analyses calculated for $\mathrm{C}_{25} \mathrm{H}_{16} \mathrm{~F}_{6} \mathrm{~N}_{2} \mathrm{~S}_{2}$ (\%): C 57.47, H 3.09, N 5.36, S 12.27; found: C 58.14, H 3.43, N 5.42, S 12.14 .

1,2-Bis(2-methyl-5-(3-quinolyl)-3-thienyl)cyclopentane (L3). L3 was prepared following the procedure described for $\mathbf{L 1}$, but using 3-bromoquinoline (1.0 mL, $7.5 \mathrm{mmol}$ ) as the bromide derivative in the Suzuki cross-coupling reaction. The crude product was then purified by column chromatography on silica gel with a 4:1 mixture of dichloromethane and ethyl acetate as the eluent, producing L3 as a bright white solid (0.87 g, 56\%).

${ }^{1} \mathrm{H}$ NMR (400 MHz, $\mathrm{CDCl}_{3}$ ): $\delta=2.10$ (s, 6H, Me), 2.15 (m, 2H, $J=7.6 \mathrm{~Hz}$, cyclopentene), 2.91 (t, 4H, $J=7.6 \mathrm{~Hz}$, cyclopentene), 7.23 (s, 2H, thiophene), $7.53\left(\mathrm{~m}, 2 \mathrm{H}, J_{1}=6.8 \mathrm{~Hz}, J_{2}=1.2 \mathrm{~Hz}\right.$, quinoline), $7.66\left(\mathrm{~m}, 2 \mathrm{H}, J_{1}=6.8 \mathrm{~Hz}, J_{2}=1.2 \mathrm{~Hz}\right.$, quinoline), 7.79 (d, 2H, $J=8.0 \mathrm{~Hz}$, quinoline), 8.07 (d, $2 \mathrm{H}, J=8.0 \mathrm{~Hz}$, quinoline), 8.14 (d, 2H, $J=2.0 \mathrm{~Hz}$, quinoline), 9.09 (d, 2H, $J=2.0 \mathrm{~Hz}$, quinoline). ${ }^{13} \mathrm{C}$ NMR (100 MHz, $\left.\mathrm{CDCl}_{3}\right): \delta=14.7,23.2,38.6,125.5,127.3,127.7,127.9,128.1$, 129.2, 129.4, 130.6, 135.0, 136.1, 136.4, 137.2, 147.2, 148.4. MS (ESI): m/z = $516.1[\mathrm{M}]^{+}$ (expected: 516.17). Elemental Analyses calculated for $\mathrm{C}_{33} \mathrm{H}_{26} \mathrm{~N}_{2} \mathrm{~S}_{2}(\%)$ : C 77.01, H 5.09, N 5.44, S 12.46; found: C 76.73, H 5.22, N 5.59, S 12.29.

1,2-Bis(2-methyl-5-(3-quinolyl)-3-thienyl)perfluorocyclopentene (L4). 5 (1.2 g, 4.0 mmol) was suspended in anhydrous THF (15 mL) under a nitrogen atmosphere. Next, $n$-buthyllithium (2.6 $\mathrm{mL}$ of a $1.6 \mathrm{M}$ solution in hexane, $4.2 \mathrm{mmol}$ ) was added dropwise at $-78{ }^{\circ} \mathrm{C}$.After stirring the 
resulting pink suspension for $30 \mathrm{~min}$, octafluorocyclopentene $(1.35 \mathrm{~mL}$ of a $20 \%$ solution in anhydrous THF, $2.0 \mathrm{mmol}$ ) was added and the reaction mixture was stirred for $1 \mathrm{~h}$ at $-78{ }^{\circ} \mathrm{C}$. Afterwards, the cooling bath was removed and the stirring continued at room temperature for $3 \mathrm{~h}$, after which dichloromethane $(25 \mathrm{~mL})$ and water $(25 \mathrm{~mL})$ were added. The organic layer was separated, washed with brine, dried over $\mathrm{Na}_{2} \mathrm{SO}_{4}$, filtered and concentrated under reduced pressure. The crude product was purified by column chromatography on silica gel using ethyl acetate as the eluent, yielding $\mathbf{L} 4$ as a pale blue solid (0.18 g, 14\%).

${ }^{1} \mathrm{H}$ NMR (400 MHz, $\mathrm{CDCl}_{3}$ ): $\delta=2.08$ (s, 6H, Me), 7.47 (s, 2H, thiophene), 7.58 (m, 2H, quinoline), 7.72 (m, 2H, quinoline), 7.84 (d, 2H, $J=8.0 \mathrm{~Hz}$, quinoline), 8.11 (d, 2H, $J=8.0 \mathrm{~Hz}$, quinoline), 8.24 (br, 2H, quinoline), 9.12 (d, $2 \mathrm{H}, J=2.0 \mathrm{~Hz}$, quinoline). ${ }^{19} \mathrm{~F}$ NMR (400 MHz, $\left.\mathrm{CDCl}_{3}\right): \delta=-131.82$ (quint, 2H, $\left.J=5.6 \mathrm{~Hz}\right),-109.97(\mathrm{t}, 4 \mathrm{H}, J=5.6 \mathrm{~Hz}) \cdot{ }^{13} \mathrm{C}$ NMR $(100 \mathrm{MHz}$, $\mathrm{CDCl}_{3}$ ): $\delta=14.8,111.1$ (multiplet), 116.2 (multiplet), 123.9, 126.3, 126.5, 127.6, 127.9, 128.0, 129.4, 129.9, 131.5, 136.4 (multiplet), 139.0, 142.7, 147.5, 148.1. MS (ESI): m/z $=623.1[\mathrm{M}]^{+}$ (expected: 623.1). Elemental Analyses calculated for $\mathrm{C}_{33} \mathrm{H}_{20} \mathrm{~F}_{6} \mathrm{~N}_{2} \mathrm{~S}_{2}$ (\%): C 63.66, H 3.24, $\mathrm{N}$ 4.50, S 10.30; found: C 63.59, H 3.70, N 4.47, S 9.76.

1,2-Bis(2-methyl-5-(5-methyl-3-pyridyl)-3-thienyl)cyclopentene (L5). L5 was prepared following the procedure described for $\mathbf{L 1}$ but using 3-bromo-5-methylpyridine (0.85 mL, 7.5 mmol) as the bromide derivative in the Suzuki cross-coupling reaction. The crude product was then purified by column chromatography on silica gel with a 3:1 mixture of ethyl acetate and dichloromethane as the eluent to yield the $\mathbf{L 5}$ as a white solid (0.69 g, 52\%).

${ }^{1} \mathrm{H}$ NMR (400 MHz, CDCl $): \delta=2.02$ (s, 6H, Me), 2.10 (m, 2H, $J=7.6 \mathrm{~Hz}$, cyclopentene), 2.34 (s, 6H, Me), 2.85 (t, 4H, $J=7.6 \mathrm{~Hz}$, cyclopentene), 7.05 (s, 2H, thiophene), 7.55 (s, 2H, pyridine), 8.29 (s, 2H, pyridine), 8.56 (s, 2H, pyridine). ${ }^{13} \mathrm{C}$ NMR (100 MHz, $\left.\mathrm{CDCl}_{3}\right): \delta=14.6,18.5,23.2$, 
38.6, 125.1, 130.0, 133.0, 133.2, 134.9, 135.7, 136.1, 137.0, 143.9, 148.7. MS (ESI): $\mathrm{m} / \mathrm{z}=444.1$

$[\mathrm{M}]^{+}$(expected: 444.17). Elemental Analyses calculated for $\mathrm{C}_{27} \mathrm{H}_{28} \mathrm{~N}_{2} \mathrm{~S}_{2}$ (\%): C 73.26, H 5.92, $\mathrm{N}$

6.33, S 14.49; found: C 72.31, H 6.17, N 6.33, S 14.09.

1,2-Bis(2-methyl-5-(1-methyl-1H-imidazol-2-yl)-3-thienyl)cyclopentane (L6). L6 was prepared following the procedure described for $\mathbf{L 1}$ but using 2-bromo-1-methyl-1H-imidazole (1.0 g, $6.2 \mathrm{mmol}$ ) as the bromide derivative in the Suzuki cross-coupling reaction. The crude product was then purified by column chromatography on silica gel with ethyl acetate (containing $1 \% \mathrm{NH}_{3}$ ) as the eluent, to yield $\mathbf{L 6}$ as a slightly pink solid ( $0.47 \mathrm{~g}, 37 \%)$.

${ }^{1} \mathrm{H}$ NMR (400 MHz, CDCl $): \delta=2.08$ (m, 2H, $J=7.6 \mathrm{~Hz}$, cyclopentene), 2.10 (s, 6H, Me), 2.83 (t, 4H, $J=7.6 \mathrm{~Hz}$, cyclopentene), 3.65 (s, 6H, N-Me), 6.84 (d, 2H, $J=1.2 \mathrm{~Hz}$, imidazole), 6.96 (s, 2H, thiophene), 7.01 (d, 2H, $J=1.2 \mathrm{~Hz}$, imidazole). ${ }^{13} \mathrm{C} \mathrm{NMR}\left(100 \mathrm{MHz}, \mathrm{CDCl}_{3}\right): \delta=14.4,23.2$, 34.6, 38.2, 122.4, 126.9, 128.5, 128.9, 135.0, 136.2, 136.2, 142.4. MS (ESI): m/z $=422.1[\mathrm{M}]^{+}$ (expected: 422.16). Elemental Analyses calculated for $\mathrm{C}_{23} \mathrm{H}_{24} \mathrm{~N}_{4} \mathrm{~S}_{2}$ (\%): C 65.68, H 5.75, N 13.32, S 15.25; found: C 65.38, H 6.07, N 13.21, S 15.43.

\section{1-(2-Methyl-5-(4-pyridiyl)-3-thienyl)-2-(2-methyl-5-phenyl-3-thienyl)-cyclopentene (L7). 6}

(0.7 g, $1.9 \mathrm{mmol})$ was dissolved in anhydrous THF $(10 \mathrm{~mL})$ under a dinitrogen atmosphere and treated with $n$-buthyllithium (1.3 mL of a $1.6 \mathrm{M}$ solution in hexane, $2.1 \mathrm{mmol})$ at room temperature. After stirring in the dark for about $45 \mathrm{~min}$, tributyl borate $(0.8 \mathrm{~mL}, 3.0 \mathrm{mmol})$ was added and the resulting bright red solution was stirred in the dark for $1 \mathrm{~h}$; it was subsequently used for the Suzuki cross-coupling reaction. Meanwhile, in a separate flask, $\left[\mathrm{Pd}\left(\mathrm{PPh}_{3}\right)_{4}\right](115 \mathrm{mg}, 5 \%$ mol) and 4-bromopyridine hydrochloride $(0.47 \mathrm{~g}, 2.4 \mathrm{mmol})$ were dissolved in a solvent mixture composed of anhydrous THF (20 mL) and $20 \%$ aqueous $\mathrm{K}_{2} \mathrm{CO}_{3}$ solution $(20 \mathrm{~mL})$ under a dinitrogen atmosphere. This two-phase system was stirred at $50{ }^{\circ} \mathrm{C}$ for 15 min and the previously 
prepared boronic derivative solution was added dropwise with a syringe. The resulting reaction mixture was then stirred in the dark overnight, after which it was cooled to room temperature and dichloromethane $(25 \mathrm{~mL})$ and water $(15 \mathrm{~mL})$ were added. The organic layer was separated, washed with brine $(25 \mathrm{~mL})$, dried over $\mathrm{Na}_{2} \mathrm{SO}_{4}$, filtered and concentrated under reduced pressure. The crude product was purified by column chromatography on silica gel with ethyl acetate as the eluent, yielding $\mathbf{L} 7$ as a brown solid (0.57 g, 72\%).

${ }^{1} \mathrm{H}$ NMR (400 MHz, $\mathrm{CDCl}_{3}$ ): $\delta=1.99$ (s, 3H, Me), 2.04 (s, 3H, Me), 2.05-2.14 (m, 2H, $J_{\text {app }}=$ $7.6 \mathrm{~Hz}$, cyclopentene), 2.85 (t, 4H, $J=7.4 \mathrm{~Hz}$, cyclopentene), 7.02 (s, 1H, thiophene), 7.20-7.25 (m, 2H, thiophene + phenyl), 7.31-7.36 (m, 4H, phenyl + pyridine), 7.48-7.51 (m, 2H, $J_{a p p}=8.4$ $\mathrm{Hz}$, phenyl), 8.52 (dd, 2H, $J=1.5 \mathrm{~Hz}, J=4.7 \mathrm{~Hz}$, pyridine). ${ }^{13} \mathrm{C}$ NMR (100 MHz, $\left.\mathrm{CDCl}_{3}\right): \delta=$ 14.6, 14.9, 23.2, 38.6, 38.6, 119.5, 124.0, 125.5, 126.9, 127.2, 129.0, 134.1, 134.5, 134.6, 135.7, 136.3, 136.6, 137.6, 137.9, 140.1, 142.0, 149.9. MS (ESI): $\mathrm{m} / \mathrm{z}=413.1[\mathrm{M}]^{+}$(expected: 413.13). $\left[\left\{\text { trans-PtCl} \mathbf{2}_{2}(\mathrm{DMSO})\right\}_{2}(\boldsymbol{\mu}-\mathrm{L} 1)\right] \quad$ (C1). $\quad$ cis $-\left[\mathrm{PtCl}_{2}(\mathrm{DMSO})_{2}\right]^{22}$ (211 mg, $\left.0.5 \mathrm{mmol}\right)$ was suspended in methanol $(40 \mathrm{~mL})$, and the system was refluxed until the complex was completely dissolved. After hot filtering the resulting yellow solution to remove any $\operatorname{Pt}(0)$ impurities, ligand L1 (104 mg, $0.25 \mathrm{mmol}$ ) was immediately added and the mixture was stirred in the dark at room temperature overnight, yielding $\mathbf{C} \mathbf{1}$ as a pale-green solid, which was filtered, washed with methanol and dried under reduced pressure (260 mg, 94\%).

${ }^{1} \mathrm{H}$ NMR (400 MHz, CDCl $): \delta=2.03$ (s, 6H, Me), 2.13 (m, 2H, $J=7.6 \mathrm{~Hz}$, cyclopentene), 2.85 (t, 4H, $J=7.6 \mathrm{~Hz}$, cyclopentene), 3.47 (s, 12H, DMSO), 7.28 (s, 2H, thiophene), 7.39 (m, 4H, $J_{\text {app }}$ $=6.4 \mathrm{~Hz}$, pyridine), 8.59 (m, 4H, $J_{\mathrm{app}}=6.4 \mathrm{~Hz}$, pyridine). ${ }^{13} \mathrm{C} \mathrm{NMR}\left(100 \mathrm{MHz}, \mathrm{CDCl}_{3}\right): \delta=15.0$, 23.0, 38.6, 44.3, 120.5, 128.9, 134.5, 135.1, 137.9, 140.8, 144.5, 151.7. MS (ESI): $\mathrm{m} / \mathrm{z}=1124.9$ 
$[\mathrm{M}+\mathrm{Na}]^{+}$(expected: 1124.94). Elemental Analyses calculated for $\mathrm{C}_{29} \mathrm{H}_{34} \mathrm{Cl}_{4} \mathrm{~N}_{2} \mathrm{O}_{2} \mathrm{Pt}_{2} \mathrm{~S}_{4}$ (\%): C 31.58, H 3.11, N 2.54, S 11.63; found: C 31.11, H 3.09, N 2.52, S 11.53.

[\{trans-PtCl $\left.\left.\mathbf{P}_{2}(\mathbf{D M S O})\right\}_{2}(\boldsymbol{\mu}-\mathbf{L} 2)\right]$ (C2). Complex $\mathbf{C 2}$ was prepared following the procedure described for the synthesis of $\mathbf{C 1}$, but using ligand $\mathbf{L} 2$ (136 $\mathrm{mg}, 0.25 \mathrm{mmol}$ ) to obtain the corresponding product as an off-white solid (295 mg, 95\%).

${ }^{1} \mathrm{H}$ NMR (400 MHz, $\mathrm{CDCl}_{3}$ ): $\delta=2.03$ (s, 6H, Me), 3.48 (s, 12H, DMSO), 7.50 (m, 4H, $J_{1}=5.6$ $\mathrm{Hz}, J_{2}=1.6 \mathrm{~Hz}$, pyridine), 7.55 (s, 2H, thiophene), 8.71 (m, 4H, $J_{1}=5.6 \mathrm{~Hz}, J_{2}=1.6 \mathrm{~Hz}$, pyridine). ${ }^{19} \mathrm{~F}$ NMR (400 MHz, CDCl $): \delta=-131.78$ (quint, $2 \mathrm{H}, J=5.6 \mathrm{~Hz}$ ), $-110.10\left(\mathrm{t}, 4 \mathrm{H}, J=5.6 \mathrm{~Hz}\right.$ ). ${ }^{13} \mathrm{C}$ NMR (100 MHz, $\left.\mathrm{CDCl}_{3}\right): \delta=15.1,44.4,121.0,126.8,127.3,137.0,143.4,146.5,152.3 . \mathrm{MS}$ (ESI): $\mathrm{m} / \mathrm{z}=1231.9[\mathrm{M}+\mathrm{Na}]^{+}$(expected: 1231.88). Elemental Analyses calculated for $\mathrm{C}_{29} \mathrm{H}_{28} \mathrm{Cl}_{4} \mathrm{~F}_{6} \mathrm{~N}_{2} \mathrm{O}_{2} \mathrm{Pt}_{2} \mathrm{~S}_{4}$ (\%): C 28.77, H 2.33, N 2.31, S 10.59; found: C 29.01, H 2.30, N 2.48, S 10.36 .

[\{trans-PtCl$\left.(\mathbf{D M S O})\}_{2}(\boldsymbol{\mu}-\mathrm{L} 3)\right]$ (C3). Complex C3 was prepared following the procedure described for the synthesis of $\mathbf{C 1}$ but using ligand $\mathbf{L 3}$ (129 $\mathrm{mg}, 0.25 \mathrm{mmol}$ ) to obtain the corresponding product as a pale-green solid (255 mg, 85\%).

${ }^{1} \mathrm{H}$ NMR (400 MHz, $\mathrm{CDCl}_{3}$ ): $\delta=2.09$ (s, 6H, Me), 2.17 (m, 2H, $J=7.6 \mathrm{~Hz}$, cyclopentene), 2.93 (t, 4H, $J=7.6 \mathrm{~Hz}$, cyclopentene), 3.47 (s, 12H, DMSO), 7.30 (s, 2H, thiophene), 7.65 (m, 2H, $J_{\text {app }}$ = 7.6 Hz, quinoline), 7.82-7.88 (m, 4H, quinoline), 8.29 (d, 2H, $J=2.0 \mathrm{~Hz}$, quinoline), 9.25 (d, 2H, $J=2.0 \mathrm{~Hz}$, quinoline), 9.30 (d, 2H, $\mathrm{J}=8.8 \mathrm{~Hz}$, quinoline). ${ }^{13} \mathrm{C}$ NMR (100 MHz, $\left.\mathrm{CDCl}_{3}\right): \delta=$ 14.9, 23.3, 38.7, 43.9, 126.8, 128.3, 128.5, 128.9, 129.2, 129.9, 131.1, 133.7, 134.2, 135.1, 137.6, 137.7, 144.5, 151.5. MS (ESI): $\mathrm{m} / \mathrm{z}=1225.0[\mathrm{M}+\mathrm{Na}]^{+}$(expected: 1224.97). Elemental Analyses calculated for $\mathrm{C}_{37} \mathrm{H}_{38} \mathrm{Cl}_{4} \mathrm{~N}_{2} \mathrm{O}_{2} \mathrm{Pt}_{2} \mathrm{~S}_{4}$ (\%): C 36.94, H 3.18, N 2.33, S 10.66; found: C 36.44, H 3.18, N 2.35, S 10.11. 
Single crystals of complex C3, suitable for X-ray diffraction studies, were obtained by slow diffusion of diethyl ether into a concentrated solution of the complex in dichloromethane.

[\{trans-PtCl $\left.\left.\mathbf{P}_{2}(\mathbf{D M S O})\right\}_{2}(\boldsymbol{\mu}-\mathrm{L} 4)\right]$ (C4). Complex $\mathbf{C 4}$ was prepared following the procedure described for the synthesis of $\mathbf{C 1}$, but using ligand $\mathbf{L} 4$ (156 mg, $0.25 \mathrm{mmol}$ ) to obtain the corresponding product as an off-white solid (260 mg, 79\%).

${ }^{1} \mathrm{H}$ NMR (400 MHz, $\mathrm{CDCl}_{3}$ ): $\delta=2.12$ (s, 6H, Me), 3.52 (s, 12H, DMSO), 7.51 (s, 2H, thiophene), $7.71\left(\mathrm{~m}, 2 \mathrm{H}, J_{\text {app }}=7.6 \mathrm{~Hz}\right.$, quinoline), 7.89-7.93 (m, 4H, quinoline), 8.39 (d, $2 \mathrm{H}, J=2.0 \mathrm{~Hz}$, quinoline), 9.27 (d, 2H, $J=2.0 \mathrm{~Hz}$, quinoline), 9.37 (d, 2H, $J=9.2 \mathrm{~Hz}$, quinoline). ${ }^{19} \mathrm{~F}$ NMR (400 MHz, $\mathrm{CDCl}_{3}$ ): $\delta=-131.84$ (quint, 2H, $\left.\mathrm{J}=5.6 \mathrm{~Hz}\right),-109.87(\mathrm{t}, 4 \mathrm{H}, J=5.6 \mathrm{~Hz}) .{ }^{13} \mathrm{C}$ NMR $(100$ $\left.\mathrm{MHz}, \mathrm{CDCl}_{3}\right): \delta=15.1,44.0,125.3,126.5,127.9,128.4,128.7,129.3,129.7,131.8,135.1,136.3$, 144.2, 145.0, 151.3. MS (ESI): $\mathrm{m} / \mathrm{z}=1332.9[\mathrm{M}+\mathrm{Na}]^{+}$(expected: 1332.92). Elemental Analyses calculated for $\mathrm{C}_{37} \mathrm{H}_{32} \mathrm{Cl}_{4} \mathrm{~F}_{6} \mathrm{~N}_{2} \mathrm{O}_{2} \mathrm{Pt}_{2} \mathrm{~S}_{4}$ (\%): C 33.90, H 2.46, N 2.14, S 9.78; found: C 33.86, H 2.47, N 2.33, S 9.64.

Single crystals of complex $\mathbf{C 4}$, suitable for X-ray diffraction studies, were obtained by slow diffusion of diethyl ether into a concentrated solution of the complex in dichloromethane.

[\{trans-PtCl $\left.\left.\mathbf{P}_{2}(\mathbf{D M S O})\right\}_{2}(\boldsymbol{\mu}-\mathbf{L} 5)\right]$ (C5). Complex $\mathbf{C 5}$ was prepared following the procedure described for the synthesis of $\mathbf{C 1}$, but using ligand $\mathbf{L} 5$ (111 $\mathrm{mg}, 0.25 \mathrm{mmol}$ ) to obtain the corresponding product as an off-white solid (235 mg, 83\%).

${ }^{1} \mathrm{H}$ NMR (400 MHz, CDCl $): \delta=2.02$ (s, 6H, Me), 2.11 (m, 2H, $J=7.6 \mathrm{~Hz}$, cyclopentene), 2.38 (s, 6H, Me), 2.84 (t, 4H, $J=7.6 \mathrm{~Hz}$, cyclopentene), 3.46 (s, 12H, DMSO), 7.10 (s, 2H, thiophene), 7.66 (s, 2H, pyridine), 8.35 (s, 2H, pyridine), 8.66 (d, 2H, $J=1.6 \mathrm{~Hz}$, pyridine). ${ }^{13} \mathrm{C}$ NMR (100 $\left.\mathrm{MHz}, \mathrm{CDCl}_{3}\right): \delta=13.6,17.5,22.0,37.4,43.2,125.5,131.3,132.2,133.9,134.8,135.1,136.2$ 136.5, 144.7, 148.4. MS (ESI): $\mathrm{m} / \mathrm{z}=1154.0[\mathrm{M}+\mathrm{Na}]^{+}$(expected: 1153.97). Elemental Analyses 
calculated for $\mathrm{C}_{31} \mathrm{H}_{38} \mathrm{Cl}_{4} \mathrm{~N}_{2} \mathrm{O}_{2} \mathrm{Pt}_{2} \mathrm{~S}_{4}$ (\%): C 32.93, H 3.39, N 2.48, S 11.34; found: C 33.03, H 3.64, N 2.62, S 11.12 .

[\{trans-PtCl $\left.\left.\mathbf{C}_{2}(\mathrm{DMSO})\right\}_{2}(\boldsymbol{\mu}-\mathrm{L6})\right]$ (C6). Complex $\mathbf{C 6}$ was prepared following the procedure described for the synthesis of $\mathbf{C 1}$, but using ligand $\mathbf{L 6}$ (105 $\mathrm{mg}, 0.25 \mathrm{mmol}$ ) to obtain the corresponding product as an off-white solid (205 mg, 74\%).

${ }^{1} \mathrm{H}$ NMR (400 MHz, CDCl $)$ ): $\delta=2.12$ (m, 2H, cyclopentene), 2.23 (s, 6H, Me), 2.89 (t, 4H, cyclopentene), 3.33 (s, 12H, DMSO), 3.68 (s, 6H, N-Me), 6.95 (d, 2H, $J=1.2 \mathrm{~Hz}$, imidazole), 7.15 (d, 2H, $J=1.2 \mathrm{~Hz}$, imidazole), 7.25 (s, 2H, thiophene). ${ }^{13} \mathrm{C}$ NMR (100 MHz, $\mathrm{CDCl}_{3}$ ): $\delta=$ 14.9, 23.2, 36.0, 38.4, 44.1, 123.1, 123.3, 128.4, 132.5, 134.9, 135.9, 139.8, 142.3. MS (ESI): m/z $=1108[\mathrm{M}]^{+}$(expected: 1107.97). Elemental Analyses calculated for $\mathrm{C}_{27} \mathrm{H}_{36} \mathrm{Cl}_{4} \mathrm{~N}_{4} \mathrm{O}_{2} \mathrm{Pt}_{2} \mathrm{~S}_{4}$ (\%): C 29.25, H 3.27, N 5.05, S 11.57; found: C 29.21, H 3.37, N 5.04, S 11.69.

trans-[PtCl $2(\mathbf{D M S O})(\mathbf{L 7})]$ (C7). Complex $\mathbf{C 7}$ was prepared following the procedure described for the synthesis of $\mathbf{C} 1$ but using equimolar amounts of cis-[ $\left.\mathrm{PtCl}_{2}(\mathrm{DMSO})_{2}\right]$ (105 mg, $\left.0.25 \mathrm{mmol}\right)$ and ligand $\mathbf{L} 7$ (103 mg, $0.25 \mathrm{mmol}$ ) to obtain the corresponding mononuclear product as a dark green solid (80 mg, 42\%).

${ }^{1} \mathrm{H}$ NMR (400 MHz, $\mathrm{CDCl}_{3}$ ): $\delta=1.97$ (s, 3H, Me), 2.07 (s, 3H, Me), 2.11 (m, 2H, 7.6 Hz, cyclopentene), 2.84 (m, 4H, cyclopentene), 3.47 (s, 6H, DMSO), 6.99 (s, 1H, thiophene), 7.24 (m, $1 \mathrm{H}, J_{1}=7.6 \mathrm{~Hz}, J_{2}=1.2 \mathrm{~Hz}$, phenyl), 7.31 (s, $1 \mathrm{H}$, thiophene), 7.34 (m, 2H, $J_{\text {app }}=7.6 \mathrm{~Hz}$, phenyl), $7.39\left(\mathrm{~m}, 2 \mathrm{H}, J_{1}=5.6 \mathrm{~Hz}, J_{2}=1.6 \mathrm{~Hz}\right.$, pyridine), 7.48 (m, 2H, $J_{\mathrm{app}}=7.6 \mathrm{~Hz}$, phenyl), 8.56 (m, 2H, $\mathrm{J} 1=5.6 \mathrm{~Hz}, \mathrm{~J} 2=1.6 \mathrm{~Hz}$, pyridine $) .{ }^{13} \mathrm{C} \mathrm{NMR}\left(100 \mathrm{MHz}, \mathrm{CDCl}_{3}\right): \delta=14.5,15.1,23.1,38.4,38.6$, 44.3, 120.5, 123.8, 125.4, 127.3, 129.0, 129.3, 133.5, 134.0, 134.3, 134.6, 136.3, 136.5, 138.4, 140.3, 141.0, 144.8, 151.7. MS (ESI): $\mathrm{m} / \mathrm{z}=780.0[\mathrm{M}+\mathrm{Na}]^{+}$(expected: 780.03). Elemental 
Analyses calculated for $\mathrm{C}_{28} \mathrm{H}_{29} \mathrm{Cl}_{2} \mathrm{NOPtS}_{3}$ (\%): C 44.38, H 3.86, N 1.85, S 12.69; found: C 43.28, H 3.94, N 2.04, S 12.46 .

$\mathbf{X}$-ray structure determination. Data for the open and closed forms of complex $\mathbf{C} 3$ and the open form of complex C4 were collected on a Bruker PHOTON100 CMOS diffractometer at beamline 11.3.1 of the Advanced Light Source (synchrotron radiation, $\lambda=0.7749 \AA$ ), respectively on a dark purple/orange (depending on crystal orientation) plate at $200 \mathrm{~K}$ and a colourless plate at $100 \mathrm{~K}$. Data reduction was done with SAINT. ${ }^{23}$ The structures were solved by intrinsic phasing using SHELXT, ${ }^{24}$ and refined by full-matrix least squares on $F^{2}$ with SHELXL-2014. ${ }^{25}$ Absorption corrections for the structure of $\mathbf{C} 4$ were done with SADABS. ${ }^{23}$ The crystal of $\mathbf{C} \mathbf{3}$ was found to contain both the open and closed forms. It is unclear if this is a consequence of co-crystallization or solid-state transformation upon exposure to light. The structure was refined as a 2-component twin using cells and twin law determined with CELL_NOW. ${ }^{26}$ Absorption corrections were done with TWINABS. ${ }^{26}$ The heavy disorder of the central part of the ligand associated with the presence of both photoisomers in the same crystal, initially defined from electron-density peaks, was refined with both 1,2- and 1,3- distances and displacement parameters restraints. Crystallographic and refinement parameters are summarized in Tables S1 and S3, for complexes C3 and C4, respectively (ESI†). All details can be found in CCDC 1565000 (C3) and 1565001 (C4) that contain the supplementary crystallographic data for this paper. These data can be obtained free of charge from the Cambridge Crystallographic Data Center via https://summary.ccdc.cam.ac.uk/structure-summary-form.

Agarose gel electrophoresis. $20 \mu \mathrm{L}$ of cacodylate-buffered solutions containing $15 \mu \mathrm{M}$ (in base pair) pBR322 plasmid DNA, 0.5-2.0 equivalents of the complexes (7.5-30 $\mu \mathrm{M})$ and 5\% DMSO were incubated in the dark for $24 \mathrm{~h}$ at $37^{\circ} \mathrm{C}$. Analogous sample solutions of free plasmid DNA 
and DNA bound to cisplatin (0.5 equiv., without DMSO) were also prepared and used as controls. After the incubation, all samples were treated with $4 \mu \mathrm{L}$ of a xylene cyanol 1x aqueous solution (containing 30\% glycerol) and subsequently electrophoretized in agarose gel (1\% in TAE buffer, Tris-Acetate-EDTA) for $1 \mathrm{~h}$ at $6.25 \mathrm{~V} \mathrm{~cm}^{-1}$, using a Bio-Rad horizontal tank connected to a Consort EV231 variable potential power supply. Next, the gel was treated with SYBR Safe DNA gel stain and subsequently photographed with a Bio-Rad Gel Doc EZ imager.

Ethidium bromide displacement assays. Samples containing $15 \mu \mathrm{M}$ (in base pair) ct-DNA and $75 \mu \mathrm{M}$ ethidium bromide in cacodylate buffer were incubated for $1 \mathrm{~h}$ at $37^{\circ} \mathrm{C}$, after which they were treated with increasing amounts of the complex stock solutions (see Materials and methods). The resulting samples, containing $1-25 \mu \mathrm{M}$ of the studied complexes and up to a $5 \%$ DMSO in a final volume of $3 \mathrm{~mL}$, were then incubated in the dark for $24 \mathrm{~h}$ at $37^{\circ} \mathrm{C}$. Following the incubation, the fluorescence emission spectra of all samples were recorded at room temperature in the range 530-800 nm, using a Horiba Jovin Yvon SPEX Fluorolog 3-22 spectrofluorometer and applying an excitation wavelength of $\lambda_{\mathrm{exc}}=514 \mathrm{~nm}$. Cacodylate-buffered solutions of ct-DNA/EB (i.e. without complex) were used as control references.

Circular dichroism spectroscopy. Samples containing $50 \mu \mathrm{M}$ ct-DNA (in base pair), 5-25 $\mu \mathrm{M}$ of the studied complexes and up to 5\% DMSO in a final volume of $3 \mathrm{~mL}$ of cacodylate-buffered solution were incubated for $24 \mathrm{~h}$ at $37^{\circ} \mathrm{C}$. Following the incubation, the circular dichroism spectra of all samples were recorded at room temperature using a Jasco J-815 CD spectropolarimeter. A wavelength range of 235-315 nm was used to minimize DMSO interference, using a bandwidth of $2 \mathrm{~nm}$, a data pitch of $0.5 \mathrm{~nm}$, scanning speed of $50 \mathrm{~nm} \mathrm{~min}{ }^{-1}$, response time of $1 \mathrm{~s}$ and 4 accumulations. Cacodylate-buffered solutions of ct-DNA were used as control references. 
Cell lines and culture. Human lung adenocarcinoma (A549), melanoma (A375), breast adenocarcinoma (MCF7), colorectal adenocarcinoma (SW620) and ovarian adenocarcinoma (SKOV3) cell lines used in this study were purchased from the American Type Culture Collection (ATCC). All cell lines were tested and authenticated by ATCC using short tandem repeat analysis and were cultured (passage number 10-25) following ATCC recommended media. A549, A375, SW620 and SKOV3 cells were cultured in DMEM medium supplemented with $10 \%$ (v/v) fetal bovine serum (FBS), 100 unit $/ \mathrm{mL}$ penicillin, $100 \mu \mathrm{g} / \mathrm{mL}$ streptomycin and $2 \mathrm{mM}$ L-glutamine. MCF7 cells were cultured in DMEM-F12 (Ham) media (1:1) supplemented with 5\% horse serum $(\mathrm{v} / \mathrm{v}), 100 \mu \mathrm{M}$ sodium pyruvate, $10 \mu \mathrm{g} / \mathrm{mL}$ insulin, $100 \mu$ unit $/ \mathrm{mL}$ penicillin, $100 \mu \mathrm{g} / \mathrm{mL}$ streptomycin and $2 \mathrm{mM}$ L-glutamine. All cell lines were grown at $37{ }^{\circ} \mathrm{C}$ under a $5 \% \mathrm{CO}_{2}$ atmosphere. The cell lines were routinely tested using a specific standard PCR to control mycoplasma contamination.

Cell viability assays. Cell proliferation was evaluated by the 3-(4,5-dimethylthiazol-2-yl)-2,5diphenyl-tetrazolium bromide (MTT) assay. Cells were plated in 96-well sterile plates at a density of $10^{5}$ cells per $\mathrm{mL}(100 \mu \mathrm{L})$ and allowed to grow for $24 \mathrm{~h}$. After attachment to the surface, the cells were then incubated with various concentrations of the studied complexes (10 and $50 \mu \mathrm{M}$ for single-point experiments and within the range 0.5-5 $\mu \mathrm{M}$ for dose-response curves), freshly dissolved in DMSO and diluted in the corresponding culture medium (DMSO final concentration $=1 \%$ ), for $48 \mathrm{~h}$ at $37^{\circ} \mathrm{C}$. Control cells were cultured in the corresponding culture medium plus the carrier $($ DMSO final concentration $=1 \%$ ). Following the treatment, $10 \mu \mathrm{M}$ MTT was added to each well for an additional $4 \mathrm{~h}$. Afterwards, the medium was aspirated, the purple formazan precipitate was dissolved in $100 \mu \mathrm{L}$ of DMSO, and the absorbance at $570 \mathrm{~nm}$ was measured in a multi-well plate reader Multiskan FC (Thermo Scientific). The cell viability was expressed as 
percentage values with respect to control cells, and the data are shown as the mean value \pm SD of three independent experiments. Dose-response curves and the corresponding $\mathrm{IC}_{50}$ values were obtained by means of non-linear regression (curve fit), calculated with the GraphPad Prism 5.0 software. For comparison purposes, the cytotoxic effect of cisplatin was also evaluated under identical experimental conditions.

\section{RESULTS AND DISCUSSION}

Preparation of the ligands and their respective platinum(II) complexes. The diarylethenebased ligands were prepared using common synthetic procedures (Figure S1). ${ }^{1,}{ }^{21}$ Ligands L1, L3 and L5-L7 were obtained in moderate to good yields, ranging from 37\% to 72\%, by Suzuki cross-coupling reaction between the in situ-generated bis-boronic ester derivative of 1,2-bis(5chloro-2-methyl-3-thienyl)cyclopentane and the corresponding bromo(hetero)arene (see Experimental section) ${ }^{28}$ Ligands $\mathbf{L} \mathbf{2}$ and $\mathbf{L} \mathbf{4}$, containing a perfluorinated cyclopentene ring, were synthesized in low yields (26\% and $14 \%$, respectively), by reaction of octafluorocyclopentene with the corresponding bromo heteroarylthiophene (see Experimental details).

The photoswitching abilities of the ligands can be assessed by UV-Vis and ${ }^{1} \mathrm{H}$ NMR spectroscopies. For example, the colorless, open form of $\mathbf{L 1}$ exhibits intense absorption bands in the UV region of the spectrum (Figures S2). The absorption observed at $\lambda=322 \mathrm{~nm}$ corresponds to the HOMO $\rightarrow$ LUMO transition, which, according to the Woodward-Hoffman rules, is required for the photocyclization process to occur. ${ }^{29} \mathrm{UV}$ Irradiation of open $\mathbf{L 1}$ gives rise to the development of an intense new absorption band in the visible region, i.e. at $\lambda=544 \mathrm{~nm}$ (Figures S2), as reflected by the deep purple color of the solution. It can be pointed out here that a 
photostationary state (PSS) is reached, which corresponds to a final equilibrium situation of the photocyclization reaction; although complete photoconversion is rarely achieved, the PSS is assumed to represent the closed form of the molecule. ${ }^{30}$ Similar features were observed for all other ligands, except for ligand L6, which cannot undergo photocyclization; this distinctive behavior was investigated theoretically, and was ascribed to the presence of a non-photoreactive HOMO-2 $\rightarrow$ LUMO transition with a higher computed oscillator strength value than that of the $\mathrm{HOMO} \rightarrow$ LUMO one, therefore impeding the photocyclization process. ${ }^{31}$ The photochemical transformation of all ligands, except L6, can also be followed by ${ }^{1} \mathrm{H}$ NMR. For instance, NMR spectra of $\mathbf{L} 2$ after different photoirradiation times are illustrated in Figure S3. These spectra are representative of the other compounds inspected in the present study (excluding $\mathbf{L 6}$ which does not cyclize). Upon UV exposure, a gradual disappearance of the original NMR signals (open form of $\mathbf{L 2}$ ) is observed, which is accompanied by the concomitant, progressive apparition of a new set of peaks ascribed to closed $\mathbf{L} 2$ (see blue asterisks in Figure S3). The upfield shift of the proton at the 4-position of the thiophene, from 7.47 to $6.85 \mathrm{ppm}$, corroborates the occurrence of the expected electrocyclic reaction, generating a new intramolecular bond at the proximal 2-position. For irradiation times longer than 5 minutes, no further variation of the peaks was noticed, therefore indicating that the PPS was reached. At this equilibrium situation, a photoconversion ratio of 0.86 could be determined from the relative intensity of the two sets of signals (i.e. from the open and closed forms of L2). Comparable features are observed for ligands L1, L3-L5 and L7.

After full characterization, platination of the ligands was carried out, generating complexes C1C7 with good yields, from $42 \%$ to $95 \%$ (Figure 1). Hence, reaction of two equivalents of cis$\left[\mathrm{PtCl}_{2}(\mathrm{DMSO})_{2}\right]$ (cis-dichlorobis(dimethyl sulfoxide)platinum(II)) with one equivalent of L1-L6 
produced the corresponding complexes C1-C6, while the reaction between one equivalent of the platinum(II) salt and one equivalent of $\mathbf{L} 7$ yielded $\mathbf{C}$.

Single crystals, suitable for X-ray diffraction studies, could be obtained for C2, C3, C4 and C6. The crystal structures of $\mathbf{C} \mathbf{2}^{1}$ and $\mathbf{C} \mathbf{6}^{31}$ were described earlier. The X-ray structures of $\mathbf{C} \mathbf{3}$ and $\mathbf{C 4}$ are reported herein. Crystallographic and refinement parameters are summarized in Tables S1 and S3 for C3 and C4, respectively (ESI $\dagger$ ), and selected coordination bond lengths and angles for $\mathbf{C} 3$ are listed in Table S2 (ESI $\dagger$ ), whereas those for $\mathbf{C} 4$ are shown in Table S4 (ESI $\dagger$ ). The molecular structures of $\mathbf{C} 3$ and $\mathbf{C} 4$ (Figures S4 and S5) are related to those of $\mathbf{C} \mathbf{2}$ and $\mathbf{C 6}$. In all cases, the platinum atom is in a square-planar environment defined by two trans chlorido ligands, an Scoordinated DMSO solvent molecule and a nitrogen atom from the heteroaromatic moiety of the ligand. It can be pointed out here that the solid-state structure of $\mathbf{C} 3$ contains both the open and closed forms of the metal complex, in a 31.6/68.4 (Figure S4), which is not commonly observed with such photoswitching molecules.

It can also be stressed that all complexes contain a DMSO, which is trans to the N-coordinated ligand (strong trans effect of S-coordinated $\mathrm{DMSO}^{32,33}$ ); in principle, this solvent molecule may be used to further functionalize the platinum compounds, ${ }^{34}$ hence increasing the versatility of the system (since numerous monodentate ligands may be used to replace this DMSO). Moreover, one may expect that the high chloride concentration in blood (95-105 mM) would convert the compounds into more hydrophilic "[$\left[\mathrm{PtLCl}_{3}\right]$ ") species (for instance a complex of the type $\left[\mathrm{PtCl}_{2}(\mathrm{DMSO}) \mathbf{L}\right]$, where $\mathbf{L}$ is phosphane ligand, was reported that could convert into $\left[\mathrm{PtCl}_{3} \mathbf{L}\right]^{-}$in the presence of $\mathrm{NBu}_{4} \mathrm{Cl}^{35}$ ), which could be hydrolyzed inside cells (with subsequent DNA binding). 
Agarose gel electrophoresis. After full characterization of complexes C1-C7, the interaction of their open and closed photoisomers with pBR322 DNA was investigated. The corresponding gel images are depicted in Figure 2. It can be mentioned here that one may expect that complexes C1C7 will exhibit a mechanism of action related to that of cisplatin. Hence, hydrolysis of the chlorides of C1-C7 should lead to the formation of aquated species, ${ }^{36,37}$ which will bind to DNA by substitution of the water molecules for purine bases. ${ }^{38}$ Due to the trans-disposition of the chlorides, it is expected that each platinum ion will mostly produce 3-intrastand cross-links and monofunctional adducts, which will undergo conversion to interstrand cross-links; ${ }^{39}$ the diplatinum complexes should therefore show strong DNA interactions. It can be pointed out that polynuclear platinum complexes (PPCs) have shown remarkable biological activities. ${ }^{40}$ Furthermore, one may anticipate that the photo-induced closing/opening of the ligand will alter the DNA-binding properties of the corresponding complex isomers. Indeed, the electronic changes taking place upon ring closure will affect the hydrolysis of the chlorido ligands (and therefore their DNA-binding aptitude), and the change of flexibility between the closed and open species will modify their DNA binding; for instance, interstrand binding may be less favorable for the closed, more rigid complexes.

As a matter of fact, for complexes C1-C5, a clearly distinct DNA-interacting behavior of the two isomeric forms is observed. At low concentration, open $\mathbf{C 1}$ appears to produce a decrease of the electrophoretic mobility of supercoiled DNA (lane 3), which may be explained by the unwinding of the double helix. However, this effect becomes less pronounced with the increase of the concentration of open $\mathbf{C 1}$ (lanes 4 to 6). In contrast to lane 3, DNA form I is detected in lanes 4-6, and the diminution of its mobility with the increase of [open $\mathbf{C 1}$ ] resembles that of supercoiled DNA treated with cisplatin (lane 2). ${ }^{41,42}$ This concentration-dependent interaction of open $\mathbf{C 1}$ with 
DNA suggests that it exhibits different binding modes, most likely arising from cooperative effects between the platinum centers and the organic ligand. It seems that with only 0.5 equiv of open $\mathbf{C 1}$, non-covalent interactions play a role (lane 3), whereas for [open $\mathbf{C 1}] \geq 1$ equiv, typical platination effects are taking place (lanes 4 to 6 ).

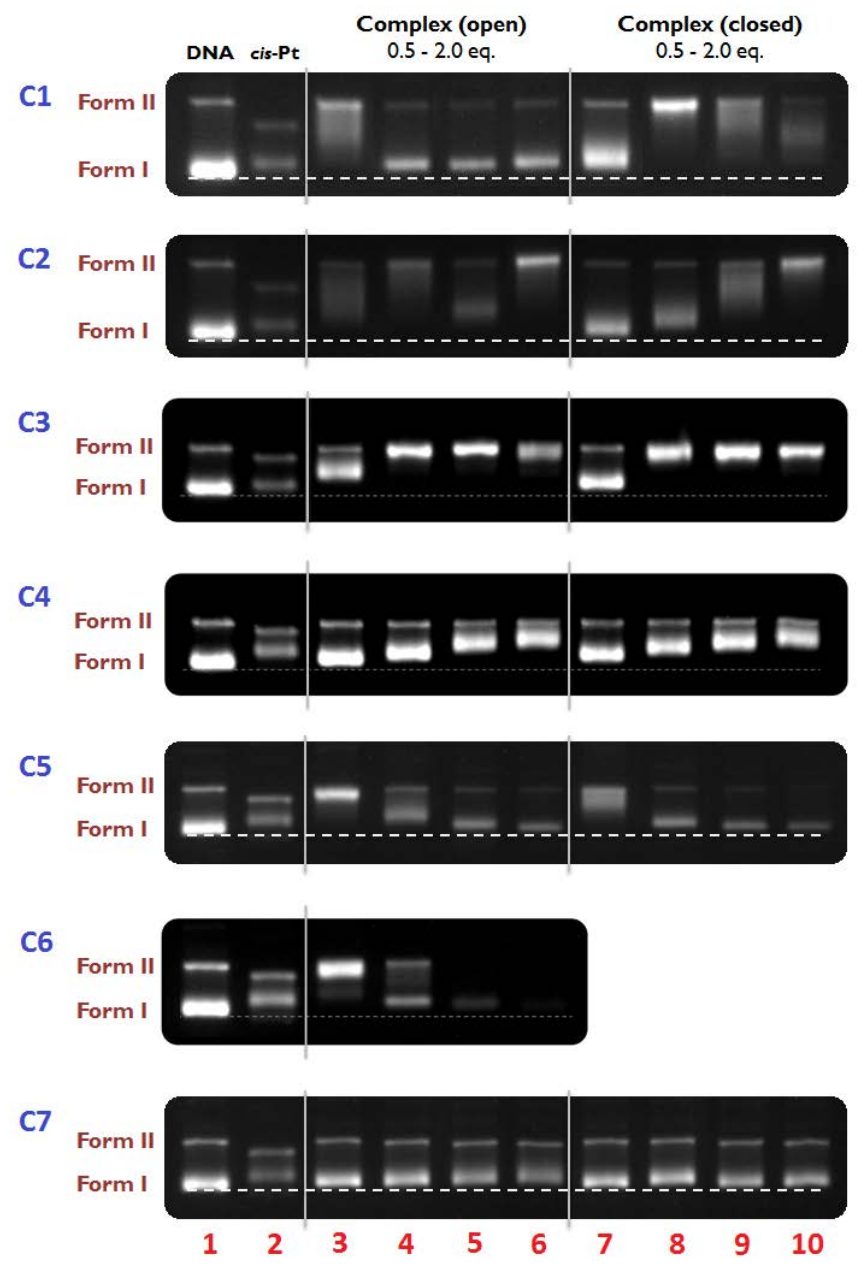

Figure 2. Agarose gel electrophoresis images for pBR322 DNA incubated for $24 \mathrm{~h}$ at $37^{\circ} \mathrm{C}$ with increasing quantities of the open and closed forms of complexes C1-C7 (solely the open form in the case of photoinactive C6). Lane 1: native plasmid DNA; lane 2: DNA + 0.5 equiv. of cisplatin; lanes 3-6: DNA + 0.5 to 2.0 equiv. of the open complex; lanes 7-10: DNA + 0.5 to 2.0 equiv. of the closed complex. 
On the contrary, closed $\mathbf{C 1}$ behaves as a strong DNA intercalator (lanes 7 to 10), probably as the result of its higher planarity and electronic conjugation. It is well known that DNA intercalation induces a stronger local unwinding of the double helix than covalent binding; this may result in the relaxation of negatively supercoiled DNA sequences until reaching an open circular conformation, whereas further addition of the intercalating agent will lead to positive supercoiling. ${ }^{43}$ Such phenomenon is clearly occurring with closed C1 (lanes 7 to 10). At lane 7, an initial decrease of the mobility is observed, as the result of a partial twist reduction of the biomolecule. Upon increasing the amount of closed $\mathbf{C 1}$, this effect is enhanced, so that all initial DNA form I is relaxed and co-migrates with DNA form II (lane 8). Upon further increase of [closed C1], the DNA mobility is improved (lanes 9 and 10), which is likely due to a subsequent induction of positive supercoiling to the previously relaxed structure (lane 8). Finally, it should be noted that a vanishing of the band intensities takes place when the amount of closed $\mathbf{C 1}$ is increased (lanes 8 to 10). This feature points to the precipitation of highly platinated DNA species.

Replacement of the cyplopentene ring by a perfluorocyclopentene one (from C1 to C2) clearly modifies the DNA-interacting properties of the resulting complex. Like open $\mathbf{C 1}$, open $\mathbf{C} 2$ also induces the unwinding of DNA, the total conversion to form II now being observed for the highest complex concentration (lane 6). This feature may be explained by single-strand cleavage of the plasmid upon platinum binding, producing the complete relaxation of supercoiled DNA fragments through free rotation around the nicked strand.

On the other hand, closed C2 exhibits intercalating properties as closed C1, but higher concentrations are required to give the same effect. Indeed, 1.0 equivalent of closed $\mathbf{C} \mathbf{1}$ is sufficient to provoke complete relaxation of supercoiled circular DNA (lane 8), whereas 2.0 equivalents are 
required with closed $\mathbf{C} 2$ (lane 10). This weaker intercalating behavior exhibited by closed $\mathbf{C} 2$ may be due to repulsive interactions between the fluorine atoms of ligand $\mathbf{L} 2$ and the phosphate backbone.

For complexes $\mathbf{C} \mathbf{3}$ and $\mathbf{C 4}$, minor differences were observed between their open and closed forms (Figure 2). In all cases, the treatment of plasmid DNA with increasing quantities of the two photoisomers of each compound gives rise to a noticeable decrease of the electrophoretic mobility of supercoiled DNA; the open and closed forms of $\mathbf{C} 3$ are even capable of inducing the comigration of supercoiled and open circular DNA (lanes 4-6 and lanes 8-10). The analogous activities of both forms of the complexes may be attributed to the larger, planar quinoline moieties of ligands $\mathbf{L} 3$ and $\mathbf{L 4}$, compared to the pyridine rings in $\mathbf{L 1}$ and $\mathbf{L 2}$, which apparently confers a comparable intercalating behavior to the photoisomers. It can also be noticed that $\mathbf{C} \mathbf{4}$ is less active than $\mathbf{C 3}$, confirming the negative influence of the fluorinated backbone of ligand $\mathbf{L} 4$ on the DNA interaction.

Open C5 follows a dose-dependent pattern that is like that of open C1 (lanes 3-6). 0.5 equivalents of open $\mathbf{C 5}$ produce the complete unwinding of supercoiled circular DNA, generating its form II (lane 3). Higher amounts of open $\mathbf{C 5}$ induce effects that are comparable to those observed with cisplatin, therefore suggesting metal binding to DNA.

Closed C5 exhibits the same behavior as its open form (see lanes 3-6 and lanes 7-10 in Figure 2). Thus, closed $\mathbf{C 5}$ does not seem to show the intercalative properties observed with the closed isomers of the previous complexes (see above). This feature may arise from (i) steric hindrance caused by the methyl substituents of the pyridine ring of ligand $\mathbf{L} \mathbf{5}$ and/or (ii) the relative disposition of the metal centers (meta position instead of para position for the previous complexes). This drastic alteration of the DNA-interacting properties of closed C5 (compared for 
instance to those of closed $\mathbf{C 1}$ and C2), triggered by a subtle modification of the metalcoordinating unit, again indicates that the binding activity of the two photoisomers proceeds via two different mechanisms.

As reported earlier, complex C6 does not display photoswitching properties. ${ }^{31}$ Consequently, only the DNA interaction of open $\mathbf{C 6}$ could be investigated. Hence open $\mathbf{C 6}$ behaves as open $\mathbf{C 1}$; namely, 0.5 equivalents of the complex lead to the formation of DNA form II (lane 3). Higher [open C6] result in effects comparable with those of the interaction of cisplatin with DNA, i.e. metal binding to the biomolecule. Actually, this covalent binding of open $\mathbf{C 6}$ appears to be particularly strong, since a vanishing of the bands is observed with the increase of its concentration (lanes 5-6), which is indicative of the precipitation of the resulting complex-DNA adducts.

The mononuclear complex $\mathbf{C 7}$ has completely distinct behavior. No photoisomeric differentiation is observed; in both cases, a comparable retardation of the DNA mobility takes place (lanes 3-6 and lanes 7-10). Surprisingly, closed C7 does not exhibit better DNA-intercalating properties than open $\mathbf{C 7}$, even though one may have expected that substitution of one of the metalbinding units by a phenyl ring (ligand $\mathbf{L 1}$ to ligand L7) would have somewhat favored the insertion of closed C7 between DNA base pairs. Consequently, it can be inferred that the strong DNAunwinding ability displayed by most closed complexes of the present study is due to not only their insertion between DNA base pairs, but also to the subsequent binding of the two platinum atoms.

Fluorescent intercalator displacement assays. Competitive binding studies were carried out subsequently, using ethidium bromide (EB) bound to ct-DNA and the open and closed forms of the complexes, as fluorescence quenchers (through the release of EB). Fluorescence spectra were therefore recorded at constant concentrations of ct-DNA and EB, respectively $15 \mu \mathrm{M}$ and $75 \mu \mathrm{M}$ ), by adding increasing amounts of both photoisomers of the platinum(II) complexes, viz. in the 
concentration range 1-25 $\mu \mathrm{M}$. Representative fluorescence spectra for open and closed $\mathbf{C} \mathbf{1}$ are shown in Figure 3. In all cases, a decrease of the fluorescent emission of the DNA/EB adduct ${ }^{44}$ is observed, which is indicative of the displacement of the dye resulting from the interaction of the complex with the double helix.
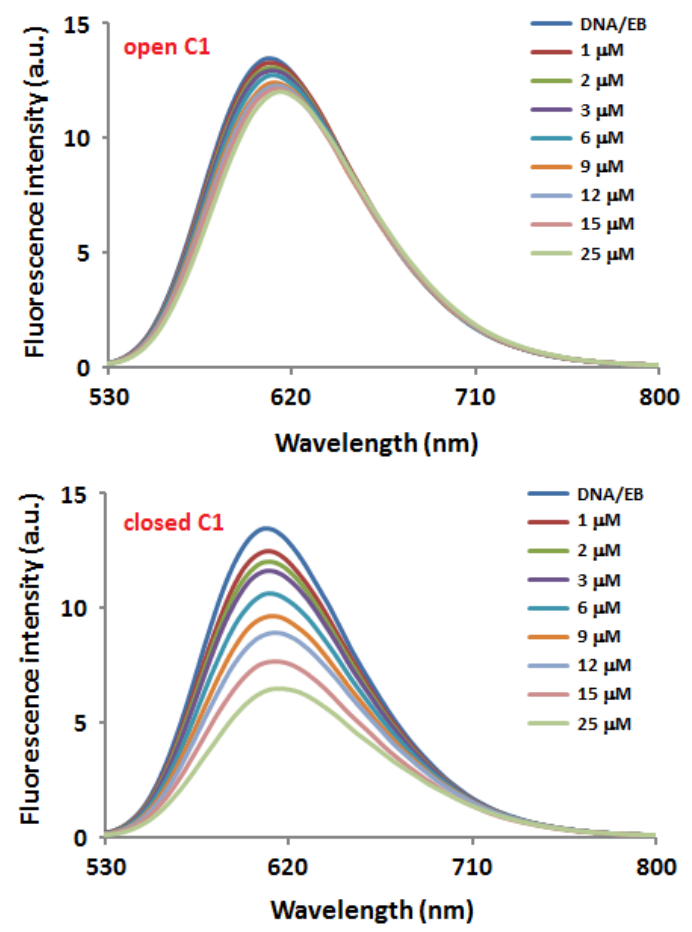

Figure 3. Fluorescent emission spectra of ct-DNA/EB samples ([DNA $]_{\mathrm{bp}}=15 \mu \mathrm{M}$, [EB] $\left.=75 \mu \mathrm{M}\right)$ treated with increasing quantities (1-25 $\mu \mathrm{M}$ ) of open $\mathbf{C 1}$ (top) and closed $\mathbf{C 1}$ (bottom), registered after incubation for $24 \mathrm{~h}$ at $37^{\circ} \mathrm{C}$.

It can be noticed that the quenching effect is clearly more pronounced for closed $\mathbf{C} \mathbf{1}$ than for open $\mathbf{C 1}$ (Figure 3), hence suggesting that the closed photoisomer has a higher capacity to expel DNA-bound EB, and thus confirming its better intercalating properties (see gel electrophoresis results) ${ }^{45}$ 
To properly compare the DNA affinity of all studied species, the fluorescence emission data obtained were used to determine the corresponding Stern-Volmer quenching constants (Ksv) for intermolecular deactivations, by applying the Stern-Volmer equation (1): ${ }^{46}$

$$
\frac{I_{0}}{I}=1+K_{S V}[Q]
$$

In this expression, $\mathrm{I}_{0}$ is the initial fluorescence intensity of the DNA/EB system, I represents the fluorescence emission intensity after the addition of a quencher, and Q is the quenching molecule, which is the metal complex in the present study. A plot of $I_{0} / I$ versus [complex] at the maximum emission wavelength (i.e. $\lambda_{\max }=610 \mathrm{~nm}$ ) gives a straight line, whose slope is equal to $K_{\text {sv }}$. Applying this procedure, Stern-Volmer constants of $K_{\mathrm{SV}}=6.6 \pm 0.8 \cdot 10^{3} \mathrm{M}^{-1}$ and $K_{\mathrm{SV}}=42.5 \pm$ $0.6 \cdot 10^{3} \mathrm{M}^{-1}$ were determined for open and closed $\mathbf{C 1}$, respectively (Figure 4).

Similar trends were observed for the open and closed photoisomers of all compounds investigated, whose respective Stern-Volmer constants are listed in Table 1.

Table 1. Calculated Stern-Volmer quenching constants for the titration of ct-DNA/EB ([DNA $]_{\mathrm{bp}}$ $=15 \mu \mathrm{M},[\mathrm{EB}]=75 \mu \mathrm{M}$ ) with the different complexes (open and closed forms), at $\lambda_{\mathrm{exc}}=514 \mathrm{~nm}$ and $\lambda_{\mathrm{em}}=610 \mathrm{~nm}$.

\begin{tabular}{|l|l|l|}
\hline Complex & $K_{\mathrm{SV}}\left(10^{3} \mathrm{M}^{-1}\right)$, open form & $K_{\mathrm{SV}}\left(10^{3} \mathrm{M}^{-1}\right)$, closed form \\
\hline C1 & $6.6 \pm 0.8$ & $42.5 \pm 0.6$ \\
\hline C2 & $4.8 \pm 0.9$ & $26.8 \pm 0.9$ \\
\hline C3 & $13.7 \pm 0.7$ & $58.0 \pm 3.0$ \\
\hline
\end{tabular}




\begin{tabular}{|l|l|l|}
\hline C4 & $18.4 \pm 0.7$ & $45.0 \pm 3.0$ \\
\hline C5 & $9.9 \pm 0.4$ & $37.4 \pm 0.8$ \\
\hline C6 $^{a}$ & $17.1 \pm 0.9$ & n/a \\
\hline C7 & $1.4 \pm 0.3$ & $21.7 \pm 0.6$ \\
\hline
\end{tabular}

${ }^{a}$ This complex does not undergo photocyclization.

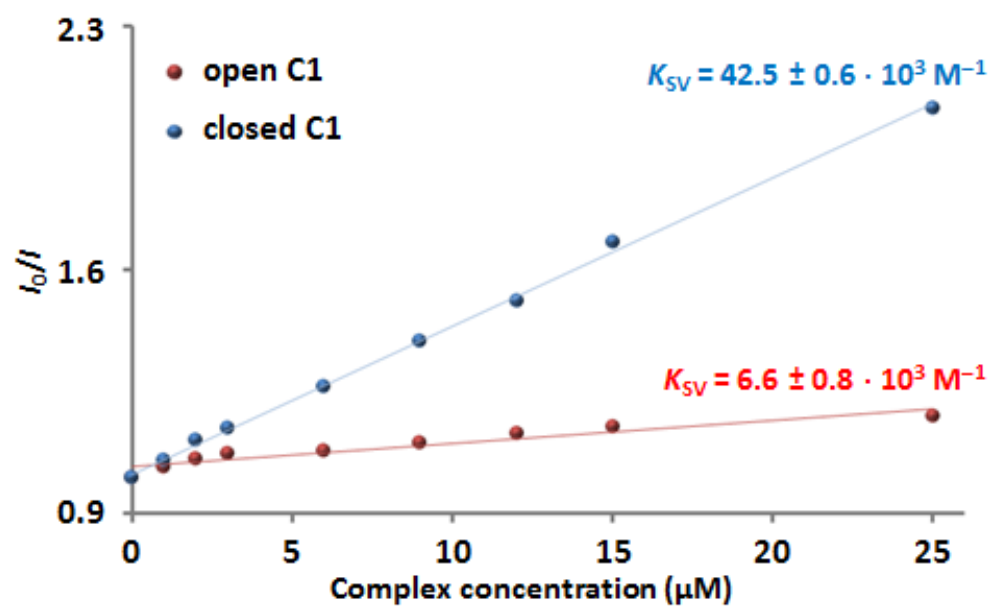

Figure 4. Plots of $\mathrm{I}_{0} / \mathrm{I}$ vs. [complex] for the titration of ct-DNA/EB samples ([DNA $]_{\mathrm{bp}}=15 \mu \mathrm{M}$, $[\mathrm{EB}]=75 \mu \mathrm{M}$ ) treated with the open (red) and closed (blue) forms of $\mathbf{C 1}$ at $\lambda_{\text {exc }}=514 \mathrm{~nm}$ and $\lambda_{\text {em }}$ $=610 \mathrm{~nm}$. Experimental data points obtained in triplicate and linear fitting of the data.

In all cases, a significantly higher $K_{\mathrm{SV}}$ value was obtained for the closed isomer, hence corroborating their higher intercalative behavior. Thus, one may expect that the closed forms of the platinum(II) complexes will induce higher growth inhibitory effects than their open counterparts. It can also be noticed that lower quenching constants were systematically achieved for the fluorinated closed-ring species, compared with their corresponding non-halogenated analogues (see $\mathbf{C 1}$ and $\mathbf{C 2}$, and C3 and C4 in Table 1). These features agree with the data achieved by gel electrophoresis (see above) and confirm the negative effect of the fluorinated cyclopentane 
ring on the intercalation of the corresponding coordination compounds. These differences may result from a slight destabilization of the DNA/complex adducts through electrostatic repulsion between the fluorine atoms and the negatively charged phosphate backbone of the double helix. ${ }^{47}$

A noticeably reduced dye-displacement ability is exhibited by the mononuclear complex $\mathbf{C 7}$ (in its both forms, see Table 1). For instance, closed $\mathbf{C 7}$ shows a $K_{\mathrm{sv}}$ value that is twice lower than that of closed $\mathbf{C 1}$. These results are in line with the earlier data obtained with plasmid DNA and confirm that the binding of dinuclear species like $\mathbf{C 1}$ to neighboring nucleobases (after the insertion of the compound between base pairs) is crucial regarding the stabilization of the resulting DNA/complex adducts.

This new set of data again show that the closed form of the diplatinum complexes are inducing stronger alterations of the double-helix conformation than the open one, apparently through a combination of intercalative and covalent interactions. It may therefore be expected that such closed species can be more efficient in inhibiting gene expression and DNA-replication processes, which may be reflected by a higher antiproliferative efficiency.

The results achieved with photoinert $\mathbf{C 6}$ requires some attention. As indicated by its $K_{\text {sv }}$ value (Table 1), this imidazolic species exhibits a far superior degree of interaction with DNA than open C1 and C2 (Table 1). Its dye-displacement activity is comparable or even greater than that of quinoline-containing complexes $\mathbf{C} \mathbf{3}$ and $\mathbf{C 4}$. Contrary to these two compounds, $\mathbf{C 6}$ does not have potentially intercalating structural motifs; hence, $\mathbf{C 6}$ can expel ethidium bromide through a different mode of action. Most likely, the binding of $\mathbf{C 6}$ strongly alters the conformation of the double helix. Local modifications of the secondary or tertiary structure of DNA, like those produced by 1,3-interstrand crosslinks, may indeed force the dye to leave the nearby interbase positions as the result of the destabilization of the $\pi$-stacking interactions. This phenomenon may 
also explain the apparent higher degree of platination and consequent band fading observed by gel electrophoresis for this complex (Figure 2).

Cell viability assays. The potential application of such metalloswitches as a novel type of photochemotherapeutic agents was examined through the evaluation of the effect of the open and closed isomers of C1-C7 (only the open form in the case of $\mathbf{C 6}$ ) on cancer cell proliferation and survivability. Five cancer cell lines were selected for these studies, namely A549 (lung adenocarcinoma), A375 (melanoma), MCF7 (breast carcinoma), SW620 (colorectal adenocarcinoma) and SKOV3 (ovarian adenocarcinoma).

In a first instance, single-point screening assays at constant complex concentrations, i.e. 10 and $50 \mu \mathrm{M}$, were performed to obtain a general comparative estimation of the cytotoxic behaviour of both photoisomers of each complex. The results achieved after an incubation time of $48 \mathrm{~h}$ are depicted in Figure 5 for complex C2, Figure 6 for $\mathbf{C 6}$ and Figure S6 for C1, C3-C5 and C7. Surprisingly (considering the DNA-binding studies), most of the screened platinum(II) complexes exhibit very poor cytotoxic properties; moreover, there is no discrimination between the activity of their open and closed isomers. Hence, treatment of the cells with open/closed C1, C3, C4 and C5 gives rise to above 75\% survival of the cell populations (Figure S6). Mononuclear C7 shows slightly better results, especially for the SKOV3 cell line, for which the closed form is more active than the open one (Figure S6). At [C7] $=10 \mu \mathrm{M}$, closed C7 can induce 50\% growth inhibition, whereas cell viability of around 95\% is observed with the open form. Much more favorable results were achieved with compound C2. Indeed, whereas the open form of this complex is not active in all tested cell lines, its closed photoisomer presents a remarkably high cytotoxic activity against most of them (Figure 5). For instance, incubation of the cells with $50 \mu \mathrm{M} \mathrm{C2}$ led to survival levels below 35\% in all cases, except for the ovarian SKOV3 line. Even more interesting is the fact that 
comparable cancer-cell death ratios were also obtained with the same cell lines using a lower amount of the complex, namely with a concentration of $10 \mu \mathrm{M}$ (Figure 5).

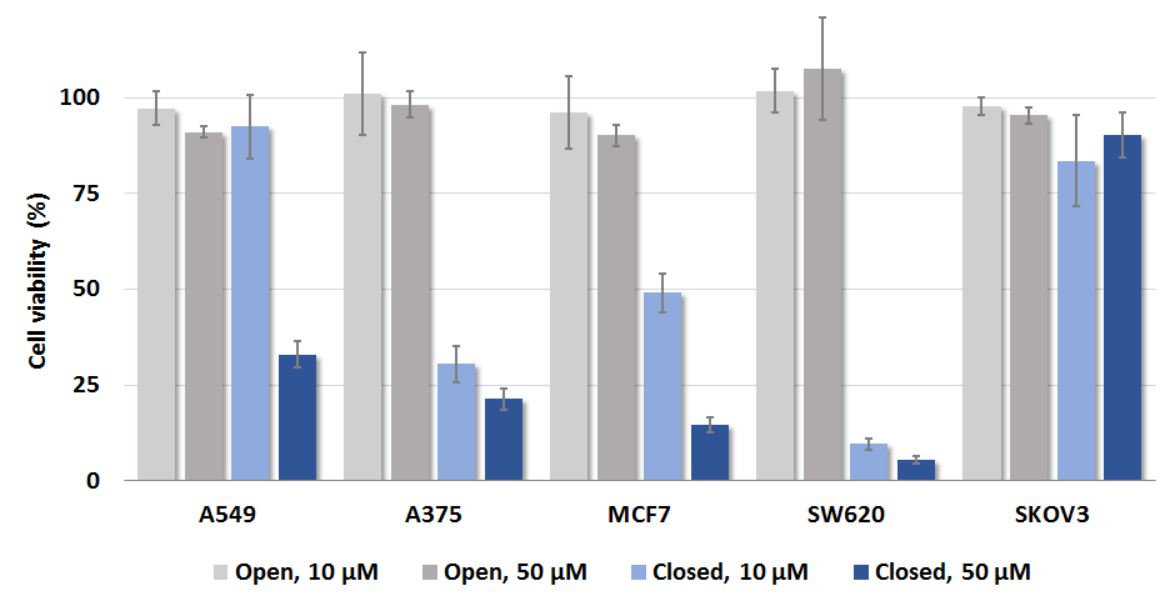

Figure 5. Single-point cell viability assays for the open and closed forms of $\mathbf{C 2}$, at constant complex concentrations (i.e. 10 and $50 \mu \mathrm{M}$ ) with incubation time of 48 hours. The results are given in \% of cell viability, using five different cancer cell lines, namely A549 (lung adenocarcinoma), A375 (melanoma), MCF7 (breast carcinoma), SW620 (colorectal adenocarcinoma) and SKOV3 (ovarian adenocarcinoma). The data shown are means \pm SD of three independent experiments.

The cell-viability results achieved with photochemically inert $\mathbf{C 6}$ were also noteworthy. In accordance with the DNA-interaction studies (see above), open $\mathbf{C 6}$ is highly cytotoxic, as reflected by the cell-death ratios of over $85 \%$ observed in all cell lines with a complex concentration of 50 $\mu \mathrm{M}$ (Figure 6). Related high antiproliferative levels were even achieved using a complex concentration of $10 \mu \mathrm{M}$, thus highlighting the different mode of action of this imidazole-based compound compared with the open forms of the pyridine- and -quinoline-containing ones (which are not cytotoxic). 
Subsequently, half-maximal inhibitory concentrations $\left(\mathrm{IC}_{50}\right)$ were determined for the two most interesting platinum(II) compounds, namely C2 and C6 (Figure S7), using the two cell lines A375 and SW620, carefully selected from the single-point results (see Figures 5 and 6) and specific characteristics. The melanoma A375 cells constitute the most suitable target for PACT drug candidates such as those herein reported, owing to the inherent accessibility to the treatment area. ${ }^{48}$ The choice of the colorectal cancer line SW620 arises from its bad prognosis and current shortfall of efficient therapies. ${ }^{49}$ The $\mathrm{IC}_{50}$ for cisplatin with these two cell lines were also determined, for comparison purposes.

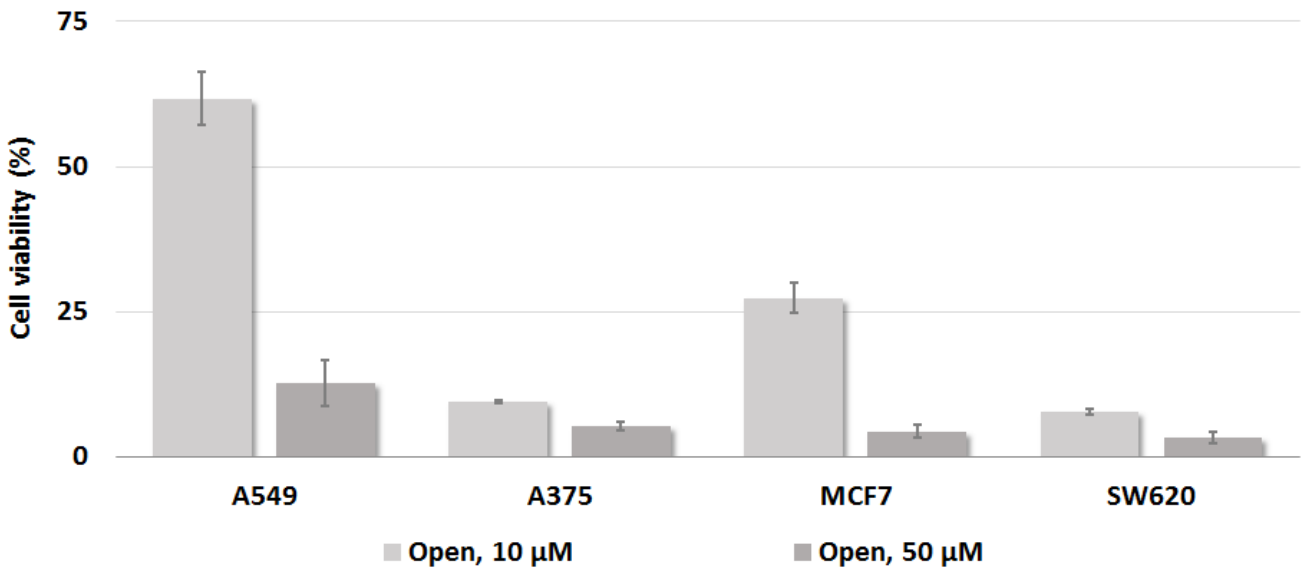

Figure 6. Single-point cell viability assays for photoinactive C6, at constant complex concentrations (i.e. 10 and $50 \mu \mathrm{M}$ ) with an incubation time of 48 hours. The results are given in $\%$ of cell viability, using five different cancer cell lines, namely A549 (lung adenocarcinoma), A375 (melanoma), MCF7 (breast carcinoma), SW620 (colorectal adenocarcinoma) and SKOV3 (ovarian adenocarcinoma). The data shown are means \pm SD of three independent experiments. 
The $\mathrm{IC}_{50}$ data listed in Table 2 corroborate the single-point cell viability assays (see above) and the cytotoxic potential of the selected compounds, which are more efficient than cisplatin for the two cell lines. The $\mathrm{IC}_{50}$ values for closed $\mathbf{C 2}$ and open $\mathbf{C 6}$ are in the low micromolar range, the compounds being up to 8 times more active than cisplatin (Table 2).

Table 2. Half-maximal inhibitory concentrations ( $\left.\mathrm{IC}_{50}, \mu \mathrm{M}\right)$ of cisplatin and complexes $\mathbf{C} 2$ and C6 for the A375 (melanoma) and SW620 (colorectal adenocarcinoma) human cell lines, after incubation for $48 \mathrm{~h}$. The results are expressed as mean values \pm SD out of three independent experiments. The dose-response curves are shown in Figure S8.

\begin{tabular}{|l|l|l|}
\hline Complex & A375 cells & SW620 cells \\
\hline Cisplatin & $6.6 \pm 0.6$ & $13.5 \pm 1.9$ \\
\hline Open C2 & $>75$ & $>75$ \\
\hline Closed C2 & $3.1 \pm 0.2$ & $2.4 \pm 0.2$ \\
\hline C6 $^{a}$ & $3.4 \pm 0.6$ & $1.8 \pm 0.3$ \\
\hline
\end{tabular}

${ }^{a}$ This complex does not undergo photocyclization.

Circular dichroism measurements. The distinct results achieved with open C6, compared with those of all other open complexes, were analyzed further by circular dichroism (CD) spectroscopy. Thus, increasing quantities of the open form of C1-C7 were added to solutions of ct-DNA, and the corresponding CD spectra were recorded in the near-UV region, viz. between 235 and 315 nm, after incubation for $24 \mathrm{~h}$ at $37^{\circ} \mathrm{C}$ (see Experimental Section for details). The spectra for $\mathbf{C 6}$ and open C1-C5 and C7 are shown in Figure 7. 

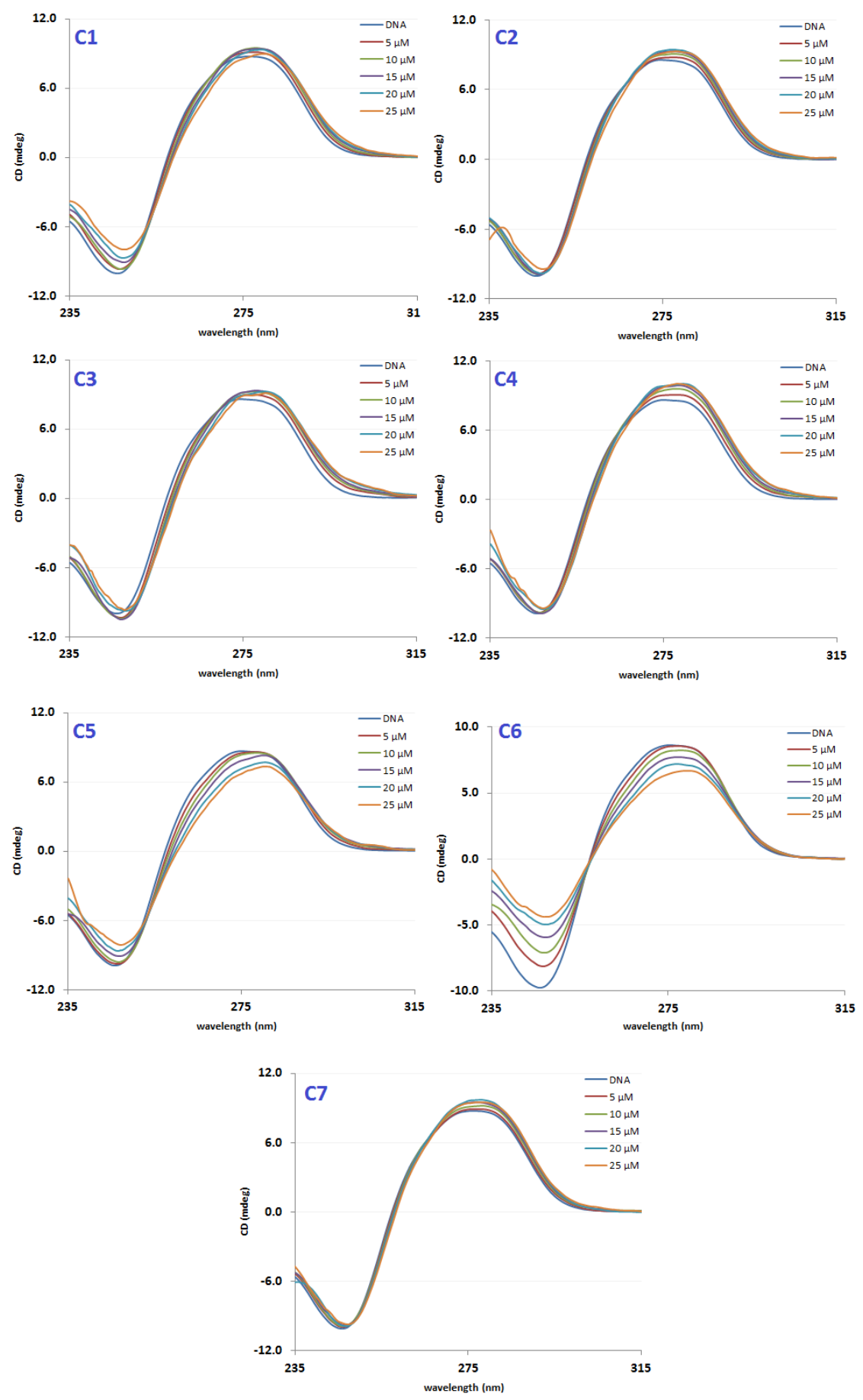

Figure 7. $\mathrm{CD}$ spectra of ct-DNA samples $\left([\mathrm{DNA}]_{\mathrm{bp}}=50 \mu \mathrm{M}\right)$ treated with increasing concentrations (5-25 $\mu \mathrm{M})$ of open complexes C1-C7, registered after incubation for $24 \mathrm{~h}$ at $37^{\circ} \mathrm{C}$. 
Direct comparison of the CD spectra of $\mathbf{C 1}$ and $\mathbf{C 6}$ interacting with B-DNA reveals noticeable differences. The addition of increasing amounts of $\mathbf{C 1}$ to ct-DNA results in a minor bathochromic shift of both the characteristic positive and negative bands, and a progressive slight increase of ellipticity of the positive signal, up to saturation. Such CD features are indicative of the monofunctional covalent binding of platinum(II) drugs to DNA, and therefore agree with the previously proposed metal-mediated activity of the open photoisomers. ${ }^{50}$ Furthermore, these spectroscopic data corroborate the minor impact of the complex binding on the helical conformation nor on the base-pair stacking of the biomolecule. Apart from C6, similar spectra were obtained for all other open complexes (see Figure 7); the spectral changes induced upon treatment of ct-DNA with the highest concentration of each complex are listed in Table S5.

Open $\mathbf{C 6}$ induces a drastically distinct alteration of the conformation of the double helix (Figure 7 and Table S5). The progressive binding of this imidazolic species leads to a severe decrease of the whole CD signal of B-DNA, particularly for the negative band. These spectral changes suggest that $\mathbf{C} \mathbf{6}$ has a distinct effect on the secondary structure of the biomolecule, most likely generating local disruptions of the helical turn, and destabilization of the alignment of adjacent base pairs. This effect is consistent with the efficient expulsion of ethidium bromide noted by fluorescence assays (see above).

Since $\mathbf{C 6}$ is the sole metal complex of the series exhibiting this behavior, it may be attributed to the methylimidazole units; however, the exact mechanism through which $\mathbf{C 6}$ induces such a distinct structural alteration of B-DNA remains to be elucidated. Probably, the specific electronic structure of ligand $\mathbf{H} \mathbf{6}^{31}$ has a direct effect on the ligand-exchange kinetics and/or the binding affinity of the platinum(II) centers, which may thus become more prone to mediate DNA cross- 
links. These different DNA-interacting properties may explain the higher cytotoxicity of $\mathbf{C 6}$, compared with that of the other open complexes (Table 2 and Figures 5, 6 and S7).

Finally, (see Figure S6)

\section{CONCLUSIONS}

Following earlier promising results, ${ }^{1}$ a series of photoswitchable platinum(II) complexes based on photoresponsive dithienylcyclopentene moieties were prepared and fully characterized. In particular, the connectivity of the metal ions was clearly established by X-ray measurements, which revealed a trans disposition of the chlorido ligands and the S-coordination of DMSO. The photochemical properties of the ligands and metal complexes prepared were investigated, which showed that the ligand $\mathbf{L} \mathbf{6}$ and the corresponding complex $\mathbf{C 6}$ were photoinactive. ${ }^{31}$

DNA-interaction studies carried out with all complexes revealed that the activity of these photoisomerisable agents can be efficiently modified through their light-mediated conversion. Clearly distinct DNA-interacting properties for the open and closed forms of the coordination compounds were observed. For instance, different metal covalent binding and ligand intercalative association were noticed for the two forms. These remarkable results suggest that the activity of each photoisomer may be tailored through appropriate structural modifications, thus opening up design possibilities for the future development of more effective DTC-based metallodrugs (DTC stands for Dithienylcyclopentene).

The antiproliferative activity of the open and closed forms of the complexes were examined, which showed that most of the compounds surprisingly were not cytotoxic; actually, the distinct DNA-interacting ability of each isomer did not translate into interesting results with cells. 
Remarkable data were though obtained with complex C2, whose closed form is highly cytotoxic against melanoma and colorectal cancer cells, whereas its open form is non-toxic. These drastically distinct behaviors of open/closed C2 further strengthen the innovative approach proposed in a previous proof of concept study, ${ }^{1}$ consisting in using DTC photoswitches to develop novel PACT agents.

\section{ASSOCIATED CONTENT}

\section{Supporting Information}

The Supporting Information is available free of charge on the ACS Publications website at DOI: 10.1021/acs.inorg-chem.xxxxxxx

Schematic representations of the structures of L1-L7; UV-vis spectra of open/closed $\mathbf{C 1} ;{ }^{1} \mathrm{H}$ NMR spectra of L2 upon UV irradiation; crystal data and structure refinement for C3 and C4; representations of the crystal structures of $\mathbf{C} 3$ and $\mathbf{C 4}$; selected bond lengths and angles for $\mathbf{C} 3$ and C4; CD data of the open complexes; CD spectra of the closed complexes; cell viability assays and dose response curves.

\section{Accession codes}

CCDC 1565000 and 1565001 contain the supplementary crystallographic data for this paper. These data can be obtained free of charge via www.ccdc.cam.ac.uk/data_request/cif, or by emailing data_request@ccdc.cam.ac.uk, or by contacting The Cambridge Crystallographic Data Centre, 12 Union Road, Cambridge CB2 1EZ, UK; fax: +44 1223336033. 


\section{AUTHOR INFORMATION}

\section{Corresponding author}

*E-mail: patrick.gamez@qi.ub.es

ORCID

Patrick Gamez: 0000-0003-2602-9525

Notes

The authors declare no competing financial interest.

\section{ACKNOWLEDGEMENTS}

Financial support from the Spanish Ministerio de Economía y Competitividad/FEDER (projects CTQ2015-70371-REDT, CTQ2014-55293-P and CTQ2017-88446-R) is acknowledged. PG thanks the Institució Catalana de Recerca i Estudis Avançats (ICREA). This work was partially supported by a grant from the Spanish government and the EU (FIS PI13/00089), a grant from La Marató de TV3 Foundation (20132730). Also, an individual contract to LKG supported by a program from the Barcelona University in collaboration with Obra Social de la Fundació Bancaria "La Caixa” is acknowledged. This research used resources of the Advanced Light Source, which is a DOE Office of Science User Facility under contract no. DE-AC02-05CH11231. 


\section{REFERENCES}

1. Presa, A.; Brissos, R. F.; Caballero, A. B.; Borilovic, I.; Korrodi-Gregorio, L.; PerezTomas, R.; Roubeau, O.; Gamez, P., Photoswitching the Cytotoxic Properties of Platinum(II) Compounds. Angew. Chem. Int. Ed. 2015, 54, 4561-4565.

2. Farrer, N. J.; Salassa, L.; Sadler, P. J., Photoactivated Chemotherapy (PACT): The Potential of Excited-state d-Block Metals in Medicine. Dalton Trans. 2009, 10690-10701.

3. Phillips, D., Light Relief: Photochemistry and Medicine. Photochem. Photobiol. Sci. 2010, 9, 1589-1596.

4. Farrer, N. J.; Sadler, P. J., Photochemotherapy: Targeted Activation of Metal Anticancer Complexes. Aust. J. Chem. 2008, 61, 669-674.

5. Benov, L., Photodynamic Therapy: Current Status and Future Directions. Med. Princ. Pract. 2015, 24, 14-28.

6. Crespy, D.; Landfester, K.; Schubert, U. S.; Schiller, A., Potential Photoactivated Metallopharmaceuticals: From Active Molecules to Supported Drugs. Chem. Commun. 2010, 46, 6651-6662.

7. Smith, N. A.; Sadler, P. J., Photoactivatable Metal Complexes: From Theory to Applications in Biotechnology and Medicine. Philos. Trans. R. Soc. A-Math. Phys. Eng. Sci. 2013, $371,13$. 
8. Doherty, R. E.; Sazanovich, I. V.; McKenzie, L. K.; Stasheuski, A. S.; Coyle, R.; Baggaley, E.; Bottomley, S.; Weinstein, J. A.; Bryant, H. E., Photodynamic Killing of Cancer Cells by a Platinum(II) Complex with Cyclometallating Ligand. Sci. Rep. 2016, 6.

9. Abadeer, N. S.; Murphy, C. J., Recent Progress in Cancer Thermal Therapy Using Gold Nanoparticles. J. Phys. Chem. C 2016, 120, 4691-4716.

10. Sun, Y. J.; Joyce, L. E.; Dickson, N. M.; Turro, C., DNA Photocleavage by An Osmium(II) Complex in the PDT Window. Chem. Commun. 2010, 46, 6759-6761.

11. Mari, C.; Pierroz, V.; Ferrari, S.; Gasser, G., Combination of Ru(II) Complexes and Light: New Frontiers in Cancer Therapy. Chem. Sci. 2015, 6, 2660-2686.

12. van Rixel, V. H. S.; Siewert, B.; Hopkins, S. L.; Askes, S. H. C.; Busemann, A.; Siegler, M. A.; Bonnet, S., Green Light-induced Apoptosis in Cancer Cells by a Tetrapyridyl Ruthenium Prodrug Offering Two Trans Coordination Sites. Chem. Sci. 2016, 7, 4922-4929.

13. Respondek, T.; Garner, R. N.; Herroon, M. K.; Podgorski, I.; Turro, C.; Kodanko, J. J., Light Activation of a Cysteine Protease Inhibitor: Caging of a Peptidomimetic Nitrile with RuII(bpy)(2). J. Am. Chem. Soc. 2011, 133, 17164-17167.

14. Albani, B. A.; Pena, B.; Leed, N. A.; de Paula, N.; Pavani, C.; Baptista, M. S.; Dunbar, K. R.; Turro, C., Marked Improvement in Photoinduced Cell Death by a New Tris-heteroleptic Complex with Dual Action: Singlet Oxygen Sensitization and Ligand Dissociation. J. Am. Chem. Soc. 2014, 136, 17095-17101. 
15. Brabec, V.; Pracharova, J.; Stepankova, J.; Sadler, P. J.; Kasparkova, J., Photo-Induced DNA Cleavage and Cytotoxicity of a Ruthenium(II) Arene Anticancer Complex. J. Inorg. Biochem. 2016, 160, 149-155.

16. Loganathan, D.; Morrison, H., Effect of Ring Methylation on the Photophysical, Photochemical and Photobiological Properties of Cis-dichlorobis(1,10phenanthroline)rhodium(III)chloride. Photochem. Photobiol. 2006, 82, 237-247.

17. Cuello-Garibo, J.-A.; James, C. C.; Siegler, M. A.; Bonnet, S., Ruthenium-Based PACT Compounds Based on an N,S Non-Toxic Ligand: A Delicate Balance Between Photoactivation and Thermal Stability. Chem. Sq. 2017, 1, 2.

18. Brieke, C.; Rohrbach, F.; Gottschalk, A.; Mayer, G.; Heckel, A., Light-Controlled Tools. Angew. Chem. Int. Ed. 2012, 51, 8446-8476.

19. Irie, M., Photochromism of Diarylethene Molecules and Crystals. Proc. Jpn. Acad. Ser. B Phys. Biol. Sci. 2010, 86, 472-483.

20. Feringa, B. L.; Browne, W. R., Molecular Switches. Second ed.; Wiley-VCH: 2011.

21. Lucas, L. N.; van Esch, J.; Kellogg, R. M.; Feringa, B. L., A New Class of Photochromic 1,2-Diarylethenes; Synthesis and Switching Properties of Bis(3-thienyl)cyclopentenes. Chem. Commun. 1998, 2313-2314.

22. Price, J. H.; Schramm, R. F.; Wayland, B. B.; Williamson, A. N., Palladium(II) and Platinum(II) Alkyl Sulfoxide Complexes - Examples of Sulfur-Bonded, Mixed Sulfur-Bonded and Oxygen-Bonded, and Totally Oxygen-Bonded Complexes. Inorg. Chem. 1972, 11, 1280-1284.

23. SADABS and SAINT, Bruker AXS Inc.: Madison, Wisconsin, USA. 
24. Sheldrick, G. M., Crystal Structure Refinement with SHELXTL. Acta Crystallogr. Sect. A 2015, 71, 3-8.

25. Sheldrick, G. M., Crystal Structure Refinement with SHELXL. Acta Crystallogr. Sect. C 2015, 71, 3-8.

26. Sheldrick, G. M. Cell_Now and Twinabs, Madison, Wisconsin, USA.

27. Hanazawa, M.; Sumiya, R.; Horikawa, Y.; Irie, M., Thermally Irreversible Photochromic Systems - Reversible Photocyclization of 1,2-Bis(2-methylbenzo[b]thiophen-3yl)perfluorocycloalkene Derivatives. J. Chem. Soc.-Chem. Commun. 1992, 206-207.

28. de Jong, J. J. D.; Lucas, L. N.; Hania, R.; Pugzlys, A.; Kellogg, R. M.; Feringa, B. L.; Duppen, K.; van Esch, J. H., Photochromic Properties of Perhydro- and Perfluorodithienylcyclopentene Molecular Switches. Eur. J. Org. Chem. 2003, 1887-1893.

29. Woodward, R. B.; Hoffmann, R., Stereochemistry of Electrocyclic Reactions. J. Am. Chem. Soc. 1965, 87, 395-397.

30. Feringa, B. L., Molecular Switches. Wiley-VCH Verlag GmbH: Weinheim - New-York Chichester - Brisbane - Singapore - Toronto, 2001.

31. Presa, A.; Barrios, L.; Cirera, J.; Korrodi-Gregorio, L.; Perez-Tomas, R.; Teat, S. J.; Gamez, P., Non-Switching 1,2-Dithienylethene-based Diplatinum(II) Complex Showing High Cytotoxicity. Inorg. Chem. 2016, 55, 5356-5364.

32. Elding, L. I.; Groning, O., Kinetics and Mechanism for Ligand Substitution-Reactions of Square-Planar (Dimethyl Sulfoxide)Platinum(II) Complexes - Stability and Reactivity Correlations. Inorg. Chem. 1978, 17, 1872-1880. 
33. Kukushkin, Y. N., Contribution of Investigations of Dimethylsulfoxide Complexes in Coordination Chemistry Theory. Koord. Khim. 1997, 23, 163-174.

34. Zheng, F.; Hutton, A. T.; van Sittert, C.; Moss, J. R.; Mapolie, S. F., Synthesis, Structural Characterization and Cis-trans Isomerization of Novel (Salicylaldiminato)platinum(II) Complexes. Dalton Trans. 2013, 42, 11163-11179.

35. Snelders, D. J. M.; Siegler, M. A.; von Chrzanowski, L. S.; Spek, A. L.; van Koten, G.; Gebbink, R., Coulombic Inter-ligand Repulsion Effects on the Pt(II) Coordination Chemistry of Oligocationic, Ammonium-functionalized Triarylphosphines. Dalton Trans. 2011, 40, 2588-2600.

36. Anderson, C. M.; Taylor, I. R.; Tibbetts, M. F.; Philpott, J.; Hu, Y. F.; Tanski, J. M., Hetero-multinuclear Ruthenium(III)/Platinum(II) Complexes That Potentially Exhibit Both Antimetastatic and Antineoplastic Properties. Inorg. Chem. 2012, 51, 12917-12924.

37. Saborit, J. M.; Caubet, A.; Brissos, R. F.; Korrodi-Gregorio, L.; Perez-Tomas, R.; Martinez, M.; Gamez, P., pH-Driven Preparation of Two Related Platinum(II) Complexes Exhibiting Distinct Cytotoxic Properties. Dalton Trans. 2017, 46, 11214-11222.

38. Baik, M. H.; Friesner, R. A.; Lippard, S. J., Theoretical Study of Cisplatin Binding to Purine Bases: Why Does Cisplatin Prefer Guanine Over Adenine? J. Am. Chem. Soc. 2003, 125, 14082-14092.

39. Johnstone, T. C.; Suntharalingam, K.; Lippard, S. J., The Next Generation of Platinum Drugs: Targeted Pt(II) Agents, Nanoparticle Delivery, and Pt(IV) Prodrugs. Chem. Rev. 2016, 116, 3436-3486. 
40. Farrell, N. P., Multi-Platinum Anti-Cancer Agents. Substitution-inert Compounds for Tumor Selectivity and New Targets. Chem. Soc. Rev. 2015, 44, 8773-8785.

41. Cohen, G. L.; Bauer, W. R.; Barton, J. K.; Lippard, S. J., Binding of Cisdichlorodiammineplatinum(II) and Trans- dichlorodiammineplatinum(II) to DNA - Evidence for Unwinding and Shortening of the Double Helix. Science 1979, 203, 1014-1016.

42. Sherman, S. E.; Lippard, S. J., Structural Aspects of Platinum Anticancer Drug-Interactions With DNA. Chem. Rev. 1987, 87, 1153-1181.

43. Bates, A. D.; Maxwell, A., DNA Topology. Oxford University Press: Oxford, 2005.

44. Meyeralmes, F. J.; Porschke, D., Mechanism of Intercalation into the DNA Duble Helix by Ethidium. Biochemistry 1993, 32, 4246-4253.

45. Dehkordi, M. N.; Lincoln, P., Comprehensive Study on the Binding of Iron Schiff Base Complex with DNA and Determining the Binding Mode. J. Fluoresc. 2013, 23, 813-821.

46. Permyakov, E. A., Luminescent Spectroscopy of Proteins. CRC Press: Boca Raton, Florida, 1993.

47. Martin-Pintado, N.; Yahyaee-Anzahaee, M.; Deleavey, G. F.; Portella, G.; Orozco, M.; Damha, M. J.; Gonzalez, C., Dramatic Effect of Furanose C2 ' Substitution on Structure and Stability: Directing the Folding of the Human Telomeric Quadruplex with a Single Fluorine Atom. J. Am. Chem. Soc. 2013, 135, 5344-5347.

48. Horbert, R.; Pinchuk, B.; Davies, P.; Alessi, D.; Peifer, C., Photoactivatable Prodrugs of Antimelanoma Agent Vemurafenib. ACS Chem. Biol. 2015, 10, 2099-2107. 
49. Aklilu, M.; Eng, C., The Current Landscape of Locally Advanced Rectal Cancer. Nat. Rev. Clin. Oncol. 2011, 8, 649-659.

50. Macquet, J. P.; Butour, J. L., Circular-Dichroism Study of DNA Platinum Complexes Differentiation Between Monofunctional, Cis-bidentate and Trans-bidentate Platinum Fixation on a Series of DNAs. Eur. J. Biochem. 1978, 83, 375-387. 
TOC

The cytotoxic properties of platinum(II) complexes can be tuned by photoisomerization of their 1,2-dithienylethene-containing ligands

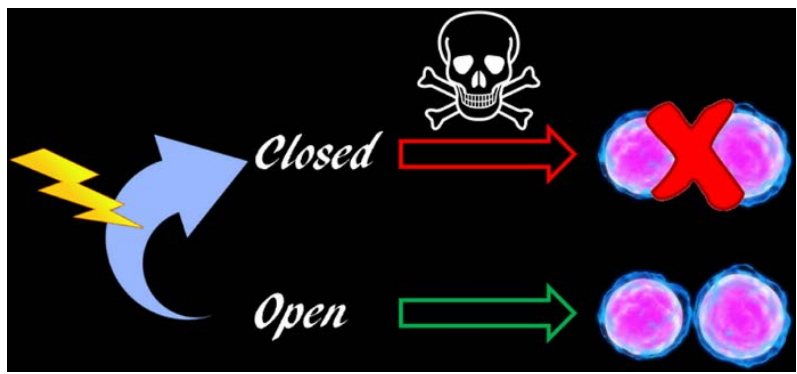

